Florida International University FIU Digital Commons

7-5-2013

\title{
Meshfree Method for Prediction of Thermal Properties of Porous Ceramic Materials
}

Maryam Zahedi

mzahe001@fiu.edu

DOI: $10.25148 /$ etd.FI13080911

Follow this and additional works at: https://digitalcommons.fiu.edu/etd

Part of the Other Mechanical Engineering Commons

\section{Recommended Citation}

Zahedi, Maryam, "Meshfree Method for Prediction of Thermal Properties of Porous Ceramic Materials" (2013). FIU Electronic Theses and Dissertations. 954.

https://digitalcommons.fiu.edu/etd/954

This work is brought to you for free and open access by the University Graduate School at FIU Digital Commons. It has been accepted for inclusion in FIU Electronic Theses and Dissertations by an authorized administrator of FIU Digital Commons. For more information, please contact dcc@fiu.edu. 


\title{
FLORIDA INTERNATIONAL UNIVERSITY
}

\author{
Miami, Florida
}

\section{MESHFREE METHOD FOR PREDICTION OF THERMAL PROPERTIES OF POROUS CERAMIC MATERIALS}

A thesis submitted in partial fulfillment of the requirements for the degree of MASTER OF SCIENCE in

MECHANICAL ENGINEERING by

Maryam Zahedi 
To: Dean Amir Mirmiran

College of Engineering and Computing

This thesis, written by Maryam Zahedi, and entitled Meshfree Method for Prediction of Thermal Properties of Porous Ceramic Materials, having been approved in respect to style and intellectual content, is referred to you for judgment.

We have read this thesis and recommend that it be approved.

$\begin{array}{r}\hline \text { Arvind Agarwal } \\ \hline \text { George Dulikravich } \\ \hline \text { Igor Tsukanov, Major Professor }\end{array}$

Date of Defense: July 5, 2013

The thesis of Maryam Zahedi is approved.

Dean Amir Mirmiran

College of Engineering and Computing

Dean Lakshmi N.Reddi

University Graduate School

Florida International University, 2013 


\section{ACKNOWLEDGMENTS}

I would like to thank Dr. Igor Tsukanov, my major advisor for his invaluable support, encouragement and guidance. His knowledge and perseverance has always been a motivation for working harder. I am profoundly grateful for all his help and dedication in teaching and guiding me always in the kindest way.

I sincerely thank my committee members Dr. George Dulikravich and Dr. Arvind Agarwal for their critical comments and suggestions they shared with me. Especially, I would like to thank Professor George Dulikravich for advising and supporting me throughout the courses of my master program. His guidance has been most helpful and appreciated.

I also, am always thankful of my valuable parents whom with their endless love, support and guidance, although from other side of the earth, made it possible for me to accomplish this research. I will be forever grateful.

My heartfelt gratitude to my true friend and husband, Reza, because of his great love, understanding, support, help and encouragement. Without him I would not have had the opportunity to do this work.

I would like to thank my lovely sister and brother for their presence in my life which always bring happiness to me.

Last but not least, I like to thank my student friends for being there when I needed, Rubel, Abbas, Siavash, Tomislav and Majid. Their help and concern were admirable. 


\title{
ABSTRACT OF THE THESIS \\ MESHFREE METHOD FOR PREDICTION OF THERMAL PROPERTIES OF \\ POROUS CERAMIC MATERIALS
}

by

\author{
Maryam Zahedi \\ Florida International University, 2013 \\ Miami, Florida \\ Professor Igor Tsukanov, Major Professor
}

In the presented thesis work, meshfree method with distance fields is applied to create a novel computational approach which enables inclusion of the realistic geometric models of the microstructure and liberates Finite Element Analysis(FEA) from the dependance on and limitations of meshing of fine microstructural feature such as splats and porosity.

Manufacturing processes of ceramics produce materials with complex porosity microstructure.Geometry of pores, their size and location substantially affect macro scale physical properties of the material. Complex structure and geometry of the pores severely limit application of modern Finite Element Analysis methods because they require construction of spatial grids (meshes) that conform to the geometric shape of the structure. As a result, there are virtually no effective tools available for predicting overall mechanical and thermal properties of porous materials based on their microstructure.

This thesis is a separate handling and controls of geometric and physical computational models that are seamlessly combined at solution run time. Using the proposed approach we will determine the effective thermal conductivity tensor of real porous ceramic materials featuring both isotropic and anisotropic thermal properties. This work involved development and implementation of numerical algorithms, data structure, and software. 


\section{TABLE OF CONTENTS}

CHAPTER

PAGE

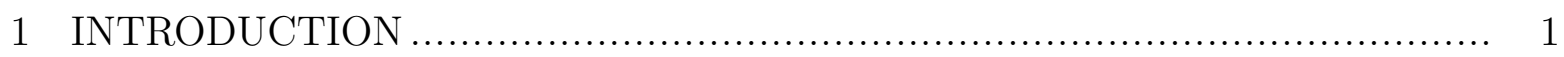

$1.1 \quad$ Problem Definition and Motivation........................................... 1

$1.2 \quad$ Objective and Research Issues.................................................. 4

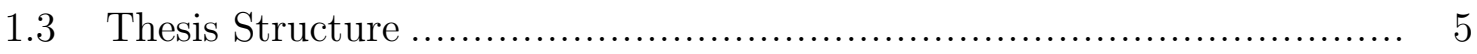

2 BACKGROUND AND LITERATURE REVIEW …................................ 6

2.1 Homogenization Methods..................................................... 6

2.1.1 Asymptotic Expansion homogenization method (AEH) …........... 8

2.2 Meshfree method with distance field ....................................... 11

2.2.1 Scan and Solve Approach ................................................ 13

3 HOMOGENIZATION OF THERMAL PROPERTIES OF POROUS CERAMIC MATERIALS USING MESHFREE METHOD WITH DISTANCE FIELD ...... 16

3.1 Asymptotic Homogenization ............................................... 16

3.2 Representative Volume Element and Periodic Boundary Condition........ 17

3.3 Solution Structure for Periodic Boundary Condition........................... 18

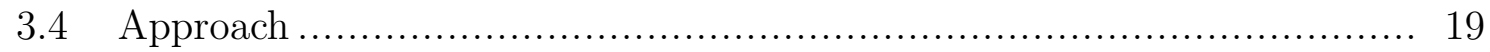

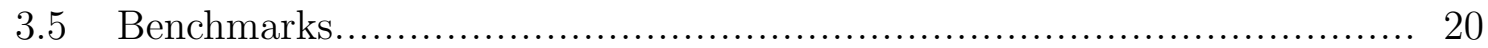

3.5.1 Test 1: Homogeneous material with no porosity .......................... 21

3.5.2 Test 2: Material with rectangular pattern of the circular holes........ 21

3.5.3 Test 3: Applying standard simulation software approach (SSSA) ..... 22

3.6 More Complicated Patterns ................................................. 27

3.6.1 Example 1: square with three diagonal circle ........................... 27

3.6.2 Example 2: square with triangle inside and holes ....................... 28

3.6.3 Example 3: square with rectangle inside ................................ 29

3.6.4 Example 4: square with ellipse inside ..................................... 30

3.6.5 Example 6: H shape ..................................................... 31

3.7 Realistic Microstructure Obtained From the Micrograph .................. 32

4 INVESTIGATING THE DEPENDENCE OF THE ANISOTROPY OF THERMAL CONDUCTIVITY ON GEOMETRIC PROPERTIES …................... 34

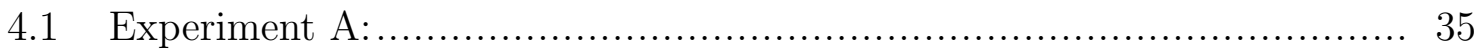

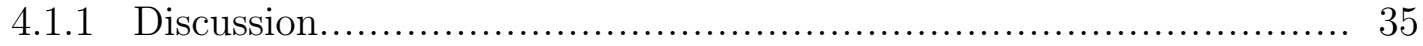

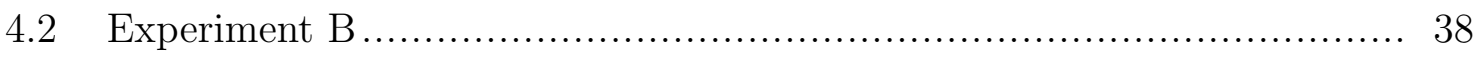

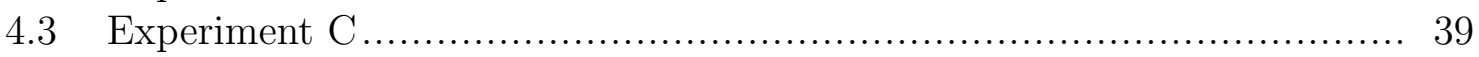

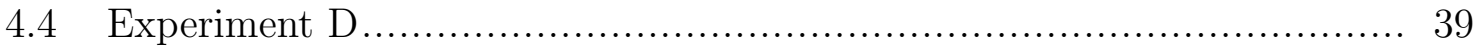

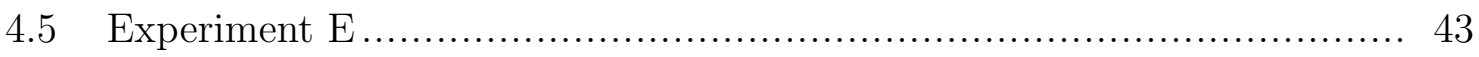

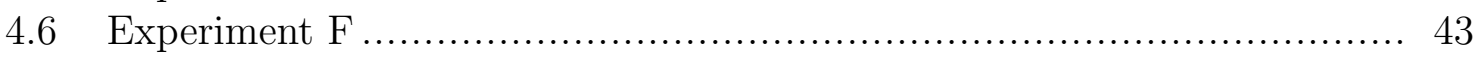

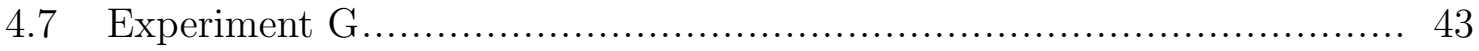

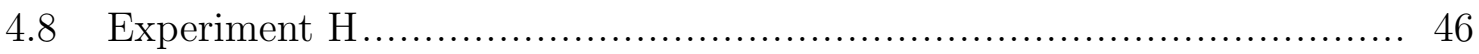

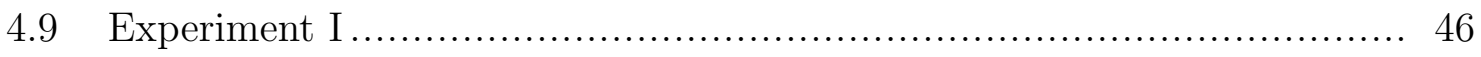

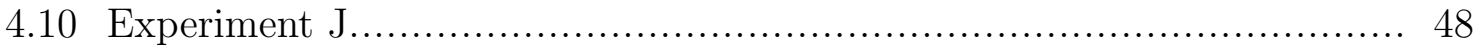

4.11 Conclusion of the Experiments ............................................... 48 


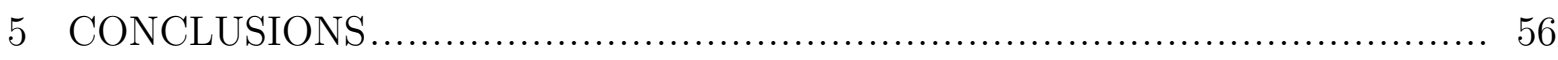

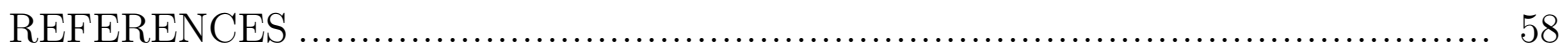




\section{LIST OF FIGURES}

FIGURE

PAGE

2.1 Representative Volume Element Selection. ................................. 7

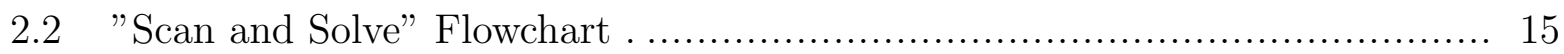

2.3 Scan\&Solve stress analysis performed from a SEM micrograph of fracture surface of $\mathrm{TaC}$ sample.

3.1 Periodic Boundary Condition

3.2 Solution structure for periodic boundary condition: (a) function $\omega$ which satisfied the homogeneous Dirichlet boundary condition; (b) shows quadratic basis function in X direction; (c) quadratic basis function in Y direction;(d) basis function of third order of polynomial in $\mathrm{X}$ direction;(e) basis function of third order of polynomial in $\mathrm{X}$ direction.

3.3 Summary of homogenization procedure

3.4 Two different RVE's and their shape functions for a material with rectangular pattern of the circular holes....

3.5 Convergence study for test 2: case one is a square with a hole in center and case two is a square with quadrant in corners....

3.6 General schematic of standard simulation packages Approach

3.7 ANSYS User Define Result Window

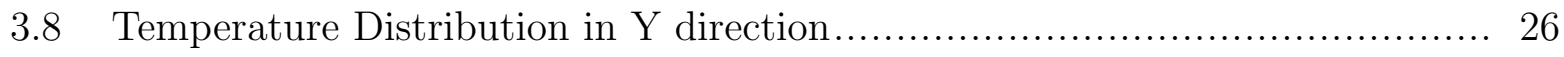

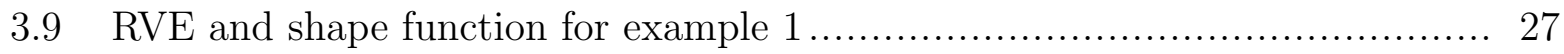

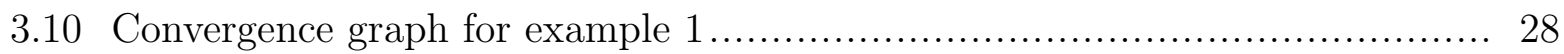

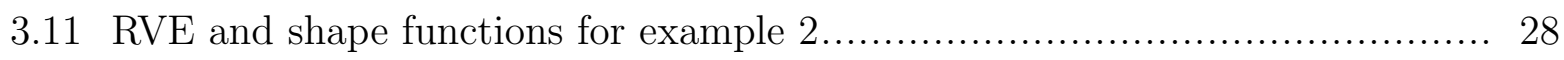

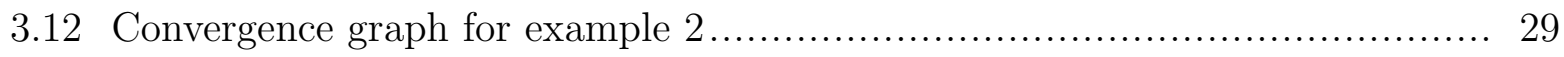

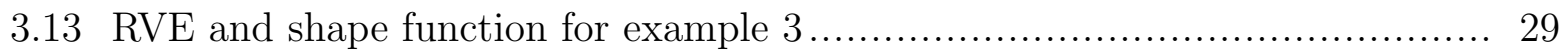

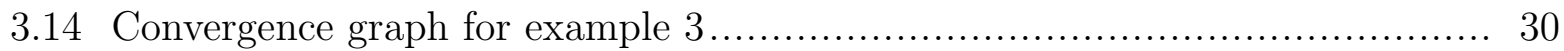

3.15 Example 4 ................................................................ 30

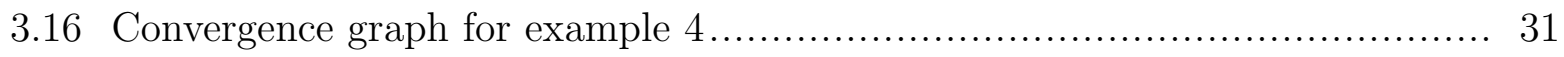

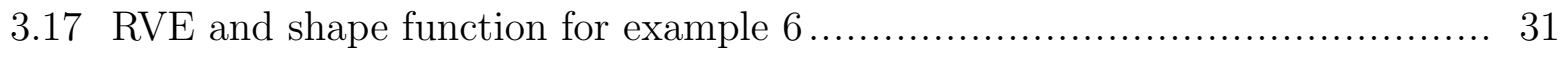


3.18 Convergence graph for example 5.

3.19 Scan\&Solve thermal analysis performed from a SEM micrograph of surface of $\mathrm{TaC}$ sample.

4.1 Cloud of porosity for experiment A, geometry of pores are ellipses ( $\mathrm{a}=0.04$, $\mathrm{b}=0.02)$ and cloud of porosity dimension is: $(0.8 \times 0.15) \ldots$

$4.2 k_{11}$ for Experiment $\mathrm{A}$, geometry of pores are ellipses $(\mathrm{a}=0.04, \mathrm{~b}=0.02)$ and cloud of porosity dimension is: $(0.8 \times 0.15)$

$4.3 k_{22}$ for Experiment $\mathrm{A}$, geometry of pores are ellipses $(\mathrm{a}=0.04, \mathrm{~b}=0.02)$ and cloud of porosity dimension is: $(0.8 \times 0.15)$

$4.4 k_{12}$ for Experiment $\mathrm{A}$, geometry of pores are ellipses $(\mathrm{a}=0.04, \mathrm{~b}=0.02)$ and cloud of porosity dimension is: $(0.8 \times 0.15)$

4.5 correlation between geometric and orthotropic axes for Experiment A, geometry of pores are ellipses $(\mathrm{a}=0.04, \mathrm{~b}=0.02)$ and cloud of porosity dimension is: $(0.8 \times 0.15)$

4.6 Cloud of porosity for experiment $\mathrm{B}$, geometry of pores are ellipses $(\mathrm{a}=0.1$, $\mathrm{b}=0.05)$ and cloud of porosity dimension is: $(0.8 \times 0.15)$

4.7 Results for Experiment B, geometry of pores are ellipses $(\mathrm{a}=0.1, \mathrm{~b}=0.05)$ and cloud of porosity dimension is: $(0.8 \times 0.15)$

4.8 Cloud of porosity for experiment $\mathrm{C}$, geometry of pores are ellipses $(\mathrm{a}=0.2$, $\mathrm{b}=0.1)$ and cloud of porosity dimension is: $(0.8 \times 0.15)$

4.9 Results for Experiment $\mathrm{C}$, geometry of pores are ellipses $(\mathrm{a}=0.2, \mathrm{~b}=0.1$ ) and cloud of porosity dimension is: $(0.8 \times 0.15)$

4.10 Cloud of porosity for experiment D, geometry of pores are ellipses $(\mathrm{a}=0.1$, $\mathrm{b}=0.05)$ and cloud of porosity dimension is: $(0.8 \times 0.4)$

4.11 Results for Experiment D, geometry of pores are ellipses $(\mathrm{a}=0.1, \mathrm{~b}=0.05)$ and cloud of porosity dimension is: $(0.8 \times 0.4)$

4.12 Cloud of porosity for experiment E, geometry of pores are ellipses $(\mathrm{a}=0.1$, $\mathrm{b}=0.05)$ and cloud of porosity dimension is: $(0.8 \times 0.6)$

4.13 Results for Experiment E, geometry of pores are ellipses $(\mathrm{a}=0.1, \mathrm{~b}=0.05)$ and cloud of porosity dimension is: $(0.8 \times 0.6)$

4.14 Cloud of porosity for experiment $\mathrm{F}$, geometry of pores are circles $(\mathrm{R}=0.02)$ and cloud of porosity dimension is: $(0.8 \times 0.15)$ 
4.15 Results for Experiment $\mathrm{F}$, geometry of pores are circles $(\mathrm{R}=0.02)$ and cloud of porosity dimension is: $(0.8 \times 0.15)$

4.16 Cloud of porosity for experiment $\mathrm{G}$, geometry of pores are circles $(\mathrm{R}=0.05)$ and cloud of porosity dimension is: $(0.8 \times 0.15)$

4.17 Results for Experiment $\mathrm{G}$, geometry of pores are circles $(\mathrm{R}=0.05)$ and cloud of porosity dimension is: $(0.8 \times 0.15)$

4.18 Cloud of porosity for experiment $\mathrm{H}$, geometry of pores are circles $(\mathrm{R}=0.1)$ and cloud of porosity dimension is: $(0.8 \times 0.15)$

4.19 Results for experiment $\mathrm{H}$, geometry of pores are circles $(\mathrm{R}=0.1)$ and cloud of porosity dimension is: $(0.8 \times 0.15)$

4.20 Cloud of porosity for experiment I, geometry of pores are circles $(\mathrm{R}=0.05)$ and cloud of porosity dimension is: $(0.8 \times 0.4)$

4.21 Results for Experiment I,geometry of pores are circles $(\mathrm{R}=0.05)$ and cloud of porosity dimension is: $(0.8 \times 0.4)$

4.22 Cloud of porosity for experiment $J$, geometry of pores are circles $(\mathrm{R}=0.05)$ and cloud of porosity dimension is: $(0.8 \times 0.6)$

4.23 Results for Experiment J, geometry of pores are circles $(\mathrm{R}=0.05)$ and cloud of porosity dimension is: $(0.8 \times 0.6)$

4.24 Comparison of the results by increasing the porosity coefficient, (a)and (b): $k_{11}$ and $k_{12}$ for porosity coefficients: 0.006, 0.018, 0.028. (c) and (d): $k_{11}$ and $k_{12}$ for porosity coefficients: $0.037,0.082,0.106$. (e) and (f): $k_{11}$ and $k_{12}$ for porosity coefficients: $0.11,0.168,0.185$.

4.25 Comparison of the results of two different pore geometry 54

4.26 Comparison of the results for different pores distribution 55 


\section{CHAPTER 1 INTRODUCTION}

\subsection{Problem Definition and Motivation}

The industrial and technological progress highly depends on creation of new materials with advanced mechanical and thermal properties. Many works are going to be done in response to the increasing need of industry to the materials with customized properties. One of these welcome materials is ceramic.

A ceramic material may be defined as any inorganic crystalline material, compounded of a metal and a non-metal. It is solid and inert. Ceramic materials are brittle, hard, strong in compression, but weak in shearing and tension. They withstand chemical erosion that occurs in an acidic or caustic environment. Ceramics generally can withstand very high temperatures such as temperatures that range from $1,000 \mathrm{C}$ to $1,600 \mathrm{C}$ $(1,800 \mathrm{~F}$ to $3,000 \mathrm{~F})$. Exceptions include inorganic materials that do not have oxygen such as silicon carbide. Glass by definition is not a ceramic because it is an amorphous solid (non-crystalline). However, glass involves several steps of the ceramic process and its mechanical properties behave similarly to ceramic materials.

Traditional ceramic raw materials include clay minerals such as kaolinite, more recent materials include aluminium oxide, more commonly known as alumina. The modern ceramic materials, which are classified as advanced ceramics, include silicon carbide and tungsten carbide. Both are valued for their abrasion resistance, and hence find use in applications such as the wear plates of crushing equipment in mining operations. Advanced ceramics are also used in the medicine, electrical and electronic industries.

The physical properties of any ceramic substance are a direct result of its crystalline structure and chemical composition. Solid state chemistry reveals the fundamental connection between microstructure and properties such as localized density variations, grain size distribution, type of porosity and second-phase content, which can all be correlated with ceramic properties such as hardness, toughness, dielectric constant, and the optical properties exhibited by transparent materials.

Mechanical properties are important in structural and building materials as well as textile fabrics. They include many properties used to describe the strength of materials 
such as: elasticity / plasticity, tensile strength, compressive strength, shear strength, fracture toughness, ductility (low in brittle materials) and indentation hardness[21].

Because of ceramic's great properties, study of them became very important. Everyday many research works are going to be conducted to improve properties of ceramic materials by changing their fabrication method and also finding the best method to measure their properties. In this research, porous ceramic materials produced by plasma spray manufacturing are under review. The final goal is to find value of thermal conductivity of this type of material.

Plasma spraying is a part of thermal spraying, a group of processes wherein a feedstock material is heated and propelled as individual particles or droplets onto a surface. The thermal spray gun generates the necessary heat by using combustible gases or an electric arc. As the materials are heated, they are changed to a plastic or molten state and are confined and accelerated by a compressed gas stream to the substrate. The particles strike the substrate, flatten, and form thin platelets (splats) that conform and adhere to the irregularities of the prepared substrate and to each other. As the sprayed particles impinge upon the surface, they cool and build up, splat by splat, into a laminar structure forming the thermal spray coating[1].

Plasma spray is the most versatile of the thermal spray processes. Plasma is capable of spraying all materials that are considered sprayable. In plasma spray devices, an arc is formed between two electrodes in a plasma forming gas, which usually consists of either argon/hydrogen or argon/helium. As the plasma gas is heated by the arc, it expands and accelerated through a shaped nozzle, creating velocities up to MACH 2. Temperatures in the arc zone approach 36,000F (20,000K). Temperatures in the plasma jet are still 18,000F $(10,000 \mathrm{~K})$ several centimeters from the exit of the nozzle. Nozzle designs and flexibility of powder injection schemes, along with the ability to generate very high process temperatures, enables plasma spraying to utilize a wide range of coatings. The range goes from low melting point polymers such as nylon, to very high temperature melting materials such as refractory materials including tungsten, tantalum, ceramic oxides, and other refractory materials. 
Hardness, density, corrosion resistance, adhesion and porosity are the characteristic of materials which used thermal spray coating for their construction. Thermal spray coatings are often used because of their high degree of hardness relative to paint coatings. Their hardness and erosion resistance make them especially valuable in high-wear applications. Such materials have high melting temperature as well as low thermal conductivity which make them very applicable to be used in power plants boilers, turbine blades, furnaces, combustion chambers and thermal coatings.

Plasma-sprayed (PS) coatings play an important role in armoring components subjected to high-heat-fluxes in different contexts. These coatings are characterized by a random distribution of porous inclusions of different size which arises from cumulative deposition and rapid solidification of partially molten droplets. Thermal barrier coatings (TBCs) for gas turbine blades are the most prominent example where high thermal isolation is required. On the other hand, PS-coatings are also considered for extreme thermo physical environments like the first wall of a nuclear fusion reactor.

Here, high energy ions and neutrons are constantly escaping the fusion plasma and hitting so called plasma-facing components (PFCs). The thermal loads are ranging from 1 to $20 \mathrm{MW} / \mathrm{m}^{2}$ in normal operation condition, while even higher peaks are expected during disruptive events. Therefore, contrary to TBC, the heat removal capability of a coating designed for PFC should be the highest possible in order to prevent melting of the involved materials and maximize the life-time of the coated components. As a result, a deep understanding of the heat-transfer mechanisms and an accurate estimation of the thermal conduction of PS-coatings is of paramount importance in the design and analysis of PFC [23].

On the other hand, we should mention that although the porosity coefficient of the plasma spray (PS) coating materials is 15 percent less than those which produced by casting method, but still existed and affects overall (homogenized) mechanical and thermal properties of the material.

Therefore, for accurate computer simulations, the effect of the material microstructure has to be accounted for. Since the size of the pores is very small $\sim 1 \mu \mathrm{m}$ a brute-force application of the engineering analysis methods based on Finite Element Method will 
result in spatial meshes with prohibitively many degrees of freedom. In fact, applicability of the existing computational tools to predict the overall mechanical and thermal properties of the realistic ceramic materials is limited by the Finite Element Method that requires construction of a spatial grid that must conform to the shape of the geometric model. In these applications, geometric model that corresponds to the material microstructure needs to be reconstructed from micrographs or /and micro CT scans.

Since the geometry of the microstructure including shape, size, orientation and volume fraction could affect the overall(homogenized) properties of the macrostructure, therefore, bringing the accurate geometry of the microstructure into consideration is of great importance. While most authors used ellipsoid or spherical pores for modeling the shape of heterogeneities, a few of them used methods which will allow them to use more realistic geometry. Only a few works are known in which people tried using realistic geometry obtained either from CT scanning or from micrographs. Construction of the spatial meshes for such geometric models is a non-trivial task which requires substantial simplification of the geometry, time consuming and error prone data transfer as human intervention.

\subsection{Objective and Research Issues}

The main goal of the proposed project is to demonstrate a feasibility of homogenization of the thermal properties of the porous ceramic materials, using meshfree method with distance fields which enables us to accommodate the realistic geometry of the microstructure into homogenization procedure. In particular, we develop numerical methods and techniques that will use meshfree method for homogenization of the thermal properties, investigate how thermal properties depend on the geometry of the microstructure as well as validate the proposed method by a number of carefully selected benchmark problems.

In this research we will introduce meshfree method and apply this new method to predict thermal properties of porous ceramic materials. Unlike other traditional simulation methods that require construction of a spatial grid (mesh) to start analyzing the properties, this research proposed a method which starts processing from the micrographs directly with no need of mesh construction, so it is called meshfree method. 
This meshfree computational technology enables seamless incorporation of the geometry into the computational model; complete automation of the solution procedure and exact treatment of all prescribed boundary conditions. Based on the distances to the geometric boundaries, this computational approach holds a promise for creating efficient multi scale and multi resolution tools that can be used to predict overall material's mechanical and thermal properties depending on its microstructure.

\subsection{Thesis Structure}

Chapter I introduces the study. First, the problem is stated. Then the research objectives and issues are explained. Chapter II provides a brief review of the history of the methods which are used in this research. The theory of homogenization methods, their benefits and drawbacks formed the first part. Second part includes the concept of meshfree method, its solution structure and equations. Chapter III describes the methodology of research, how Asymptotic Homogenization is combined with meshfree method to get the result.It also includes a number of numerical experiments to validate the proposed approach. Chapter IV presents a series of targeted numerical tests to investigate the dependence of the homogenized thermal properties on the geometry of the microstructure. We used the results to form comparison graphs, in order to see the differences more clearly. 
CHAPTER 2

\section{BACKGROUND AND LITERATURE REVIEW}

\subsection{Homogenization Methods}

The effective properties of macroscopic homogeneous composite materials can be derived from the microscopic heterogeneous material structures using homogenization techniques. Homogenization is a technique for macro micro-scale transition. In a twoscale method two spatial variables are introduced: $x$ is a macroscopic spatial coordinate and $y=x / \varepsilon$ is a microscopic one. The variable $y$ is associated with the small length scale of the inclusions or heterogeneities. The two-scales process introduced in the partial differential equations of the problem produces equations in $\mathrm{x}, \mathrm{y}$ and both variables. Generally speaking, equations in y are "solvable" if the microscopic structure is periodic, and this leads to a "rigorous" deduction of the macroscopic equations (in x) for the global behavior.

In most problems, a mathematical proof of the convergence of solutions to the "homogenized solutions" is available when $\epsilon \rightarrow 0$. It should be noticed that the "homogenized coefficients" only depend on the local (or microscopic) structure of the medium, and may be obtained by numerical solution of some boundary value problems in a period of the structure, the boundary conditions being mostly of the periodic type. In fact, homogenization gives relevant information on the relation between the local and global behaviors; for most problems in mechanics the micro and macro-processes are of very different nature.

The macroscopic mechanical properties of composite material is described by its microstructure behavior which is exemplified by the interaction between the constituents. Many heterogeneous materials have regular microstructure which makes it possible to consider only one small periodic element of the structure - representative volume element (RVE). (see Fig 2.1).

All computations are performed over RVE, and then, they are extended to the whole material. As a general procedure it could be said that in most homogenization methods a local problem of a single inclusion is solved to get the approximation of the local field behavior,(as it was done by Eshelby in 1957 for elastic fields of an ellipsoidal inclusion) 


\begin{tabular}{lllll}
0 & 0 & 0 & 0 & 0 \\
0 & 0 & 0 & 0 & 0 \\
0 & 0 & 0 & 0 & 0 \\
0 & 0 & 0 & 0 & 0 \\
0 & 0 & 0 & 0 & 0 \\
\hline
\end{tabular}

(a)

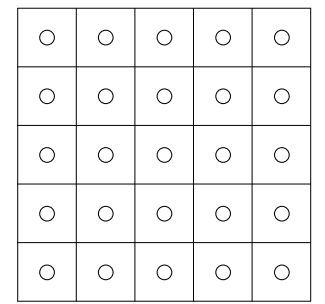

(b)

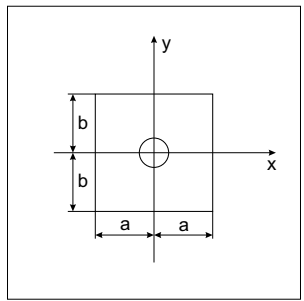

(c)

Figure 2.1: Representative Volume Element Selection.

then, by averaging these local fields, we could find global properties. This is called basic mean field homogenization method. Hashin Shtrikman type bounds, Mori-Tanaka type models and classical self schemes are the most famous homogenization methods of this type.

In all of these methods, composite is restricted to the matrix-inclusion type with perfect interfacial bonds between inclusions and their immediate surrounding matrix and differences between methods related to the assumption which is made to treat with these interaction. The Mori-Tanaka method approximates the interaction between the phases by assuming that each inclusion is embedded in an infinite matrix which is remotely loaded by the average matrix strain or average matrix stress.

Hashin and Shtrikman uses variational bounding techniques to obtain more useful estimate of modula for isotropic heterogenous materials. They assumed that the particles are spherical and the action of whole heterogenous material on any inclusion is transmitted through a spherical shell, which lies wholly in the matrix [9]. According to [8], it can be seen that the upper Hashin-Shtrikman bound corresponds to the MoriTanaka result. The upper bound can also be obtained with the Mori-Tanaka method just by interchanging matrix and inclusion material.

Lielens method is another homogenization method which uses the advantages of Mori-Tanaka method for a two phase material. This method is a properly chosen interpolation between the Mori-Tanaka and inverse Mori-Tanaka method and also between the Hashin-Shtrikman bounds, respectively. The self-consistent method approximates the interaction between phases by assuming that each phase is an inclusion embedded in a homogenous medium that has the overall properties of the composite. Therefore 
the equation will be implicit and nonlinear. In general, the self-consistent method gives a sufficient prediction of the behavior of polycrystal but it is less accurate in the case of two-phase composites[8].

Also, the "Effective Self-Consistent Scheme (ESCS)" and "Interaction Direct Derivative (IDD)" are the most recent homogenization method, proposed by Zheng and Du(2001) based on three-phase model. In the three-phase model, the inclusion is embedded in a matrix which itself embedded in an unbound, initially unknown effective medium. The ESCS overcomes the restrictions on spherical and cylindrical inclusions but still has a complicated structure. Interaction Direct Derivative (IDD)method is a simplified and explicit version of the ESCS method and has a very simple structure with clear physical meaning of the single constituent parts[8].

\subsubsection{Asymptotic Expansion homogenization method (AEH)}

Asymptotic expansion homogenization (AEH) method is another approach for multi-scale problems. This method is a very good methodology to model physical phenomena in heterogenous material with periodic microstructure. The method of asymptotic homogenization proceeds by introducing the fast variable $\xi=x / \epsilon$ and posing a formal expansion in $\epsilon$ :

$$
u(x)=u(x, \xi)=u_{0}(x, \xi)+\epsilon u_{1}(x, \xi)+\epsilon^{2} u_{2}(x, \xi)+O\left(\epsilon^{3}\right)
$$

According to [22], we can introduce a recipe for this type of homogenization as following:

- Step 1. Modeling: the scale is modeled with any Simulation software (mostly FEM) and define the $X^{e}$ as global and $Y^{e}$ as local variables.

- Step 2. Introduce the asymptotic series approximation in $\epsilon$ : AEH is a perturbation technique based on an asymptotic series expansion in $\epsilon$, a scale parameter, of a primary variable such as displacement. The first two terms of the series, represent the sum of global terms and oscillating small scale term. As mentioned earlier, $\epsilon$ 
is a scale parameter which is a kind of ratio between micro and macro scale. We could refer to smooth global term as $u^{0}$ and the small local term as $u^{1}$.

- Step 3. Derivation of hierarchical equations: this step is problem dependant. In other words it can be different for elasticity, plasticity or heat conduction problems.

- Step 4. Micro equation: derivation equation in step 4 , results in partial differential equation in $u^{1}$ to $u^{0}$. Instead of solving the equation directly for $u^{1}$ a characteristic function will be used. This function can be obtained by solving an auxiliary equation with any numerical method (FEM for example). The characteristic function which could also be called corrector, is used to relate $u^{1}$ to $(\partial u)^{0} / \partial x$ either through a numerical or an analytical solution.

- Step 5. Homogenization: the gradient of the corrector is used to define a homogenized property tensor in $Y^{e}$.

- Step 6. Solve the global boundary value problem: by the use of any method such as FEM and characteristic function from step 4, the field equation can be solved in $X^{e}$. This gives the globally smooth solution $u^{0}$.

- Step 7. Localization: at last the localization equations will be derived indirectly from step 3.

Up to now, different methods of homogenization were introduced. There are more mathematical details about these methods that could be found in [8] and [9] and [22]. Also, there are bunch of papers about application of these methods in structural analysis which used constitutive equations for their calculation which are out of scope of this research so they are ignored. In this research, we are considering homogenized thermal conductivity of the heterogeneous composite material. Unlike solid mechanic problems in composite materials, only a few works have been done in thermo-fluid area which in the following some of them are mentioned.

In [19] Mori-Tanaka method is applied for finding effective thermal conductivity of the composite media reinforced with ellipsoidal inclusions, then extended to account 
for random orientation of particles and particle size distribution. Comparison of experimental and numerical results demonstrated that Mori-Tanaka method is still applicable for these complex systems.

In [7] authors used both Hashin-Shtrikman bounds and Effective Medium Theory (EMT) to find thermal conductivity of porous materials. According to them isotropic materials which presented as 'porous' may be divided into two classes: internal porosity material in which the optimal heat transfer pathway is through the continuous phase like granular or particulate materials, in which the void volume may be occupied by either liquid or gaseous components and external porosity materials are those in which the optimal heat transfer pathway is through the dispersed phase. It may refer to a material having a continuous solid matrix that contains pores/bubbles, which may be isolated or interconnected. They believed that a model that accurately predicts the effective thermal conductivity of internal porosity will not necessarily be applicable to external porosity materials or vice versa. Their proposed bounds support conclusions from previous studies that suggested there was inherently greater uncertainty involved with predicting the effective thermal conductivity of external porosity materials than there is with internal porosity materials.

Among different homogenization methods, Asymptotic Expansion is more often used for modeling the thermal conductivity in heterogenous material. Young Seok Song and Jae Ryoun Youn examined the effective thermal conductivity tensor of carbonnano-tubes (CNT) filled composites by using Asymptotic Expansion Homogenization method(AEH) [18]. According to them this method is able to perform both localization and homogenization for the heterogenous medium. In multi-scale approach, the homogenization and the localization are the main concerns: the former yields smeared material properties used in the macroscopic field equations and the latter provides estimation of the microscopic material behaviour based on the macroscopic solution and this is one of the superiorities over other methods. Also contribution of complex geometries and anisotropic material properties of fillers can be precisely calculated through AEH method which can not be handled by other analytic models. Yasser M.Shabana and Naotake Noda in [10] used AEH to evaluate the thermomechanical effective properties of another 
group of heterogenous material which is called Functionally Graded Material (FGM) . We will also use Asymptotic Expansion Homogenization method to predict the thermal conductivity of the composite ceramic materials.

\subsection{Meshfree method with distance field}

In all of the aformentioned homogenization methods, one thing was in common and it was the usage of Finite Element Method (FEM) in discritizing the domain. The Finite Element Method (FEM) has become one of the most popular and powerful analytical tools for studying the behavior of a wide range of engineering and physical problems. Quick development of several general- purpose finite element software packages which verified and calibrated over the years made them available almost to everyone who asks and pays for them[17].

In applied mathematics, finite element method (FEM) is a general mathematical tool for obtaining approximate solutions to boundary value problems. It uses variational methods (the Calculus of variation) to minimize an error function and produce a stable solution.

Same as the idea of approximating the larger circle by many tiny straight lines, FEM also approximates a complex equation on a large domain by many simple equations on smaller sub-domain called finite elements. Applying finite element method dates back to long times ago. For instance, finding the circumference of a circle by approximating it by the perimeter of polygon by ancient mathematicians was among first FEM applications [14] The basic ideas of the finite element method as known today were presented in the papers of Turner et al., [20] and Argyris et al., [2].

Application of finite element method was quickened by super-fast development of high speed digital computers. The book by Przemieniecki [11] presents the finite element method as applied to the solution stress analysis problem. Zienkiewich et al.,[3] presented the broad interpretation of the method and its applicability to any general field problem.

Although FEM based methods were considered as a revolution in computational analysis, but it has some shortcomings in finding the physical properties from the realistic geometry of materials. In this regard, it is required to construct a spatial grid that must 
conform to the shape of the geometric model which is taken from the micro graphs. It will be very difficult due to the fact that meshing could not be done precisely for this kind of geometries. As a result, to avoid difficulties of mesh construction, meshfree method is applied.

At the core of the meshfree method of analysis with distances field is representation of a physical field by the Taylor series expansion, originally proposed by Kantorovich[6] and developed by Rvachev [12] and [13].

$$
u(w)=u(0)+\sum_{k=1}^{m} \frac{1}{k !} u_{k}(0) \omega^{k}+\omega^{m+1} \Phi
$$

This representation is a straightforward generalization of a classical Taylor series, where the term $\left|x-x_{0}\right|$ measuring the distance to the point $x_{0}$, is replaced by $\omega$ measuring the distance to a set of points. Similarly, the $k^{\text {th }}$ order derivatives of the function $u$ in the classical Taylor series are replaced by coefficients $u_{k}$ that are $k^{t h}$ order derivatives of the function $u$ in the direction $n$ normal to the boundary of a geometric domain. In contrast to classical Taylor series, where the coefficients are constants, $u_{k}(x, y, z)$ in the expression (2.2) may be arbitrary functions. This also holds when $\omega$ represents approximate distance to the geometric boundary.

Taylor series (2.2) provides connection between the values of a physical field at any spatial point and values of the field and its normal derivatives prescribed on the boundary of a geometric domain. In the context of engineering analysis this means that the function $u$ given by expression (2.2) satisfies specified boundary conditions exactly. The remainder term $\omega^{m+1} \phi$ assures completeness of the Taylor series (2.2), and it can be used to satisfy additional constraints imposed on $u$, which are usually formulated in the form of differential equations, integral equations, or variational principles.

To find a function $u$ that satisfies both boundary conditions and additional constraints one needs to determine the function $\phi$. In most cases, this problem has no exact solution. Thus, $\phi$ is approximated by linear combination of basis functions:

$$
\phi=\sum_{i=1}^{N} C_{i} \chi_{i}
$$


Now, the solution of the original problem is transformed into determining the numerical values of the coefficients $C_{i}$ in expression (2.3) by any standard numerical method. The basis functions $\chi_{i}$ in the last expression can be chosen from any sufficiently complete system of linearly independent functions: polynomials, radial basis functions, B-splines or even finite elements. Representing physical fields by Taylor series (2.2) reveals two salient features of our meshfree method: exact treatment of boundary conditions (this is the only meshfree method which allows exact treatment of different types of boundary conditions), and clean and modular separation of geometric and analytic information [16].

The shape of the geometric domain is completely described by distance $\omega$ to the boundary; and the basis functions can be defined on a grid that does not conform to the geometric input. Since a physical field u represented by expression (2.2) satisfies the prescribed boundary conditions exactly, the solution procedure needs to determine numerical values of the coefficients $C_{i}$ in the remainder term (2.3) such that $\mathrm{u}$ gives the best approximation to the differential equation of the problem.

A typical solution procedure includes construction of distance fields to the boundaries where boundary conditions are specified, differentiation of the functions in the Taylor series (2.2) with respect to spatial coordinates, integration over the un-meshed geometric domain and its boundary, solution of an algebraic problem, and visualization of the analysis results.

\subsubsection{Scan and Solve Approach}

The use of distance fields derived from sampled data makes it possible to implement a scan-and-solve approach to modeling of physical fields, which is particularly effective when physical fields need to be modeled and analyzed in existing artifacts for which traditional geometric models may not exist. Reverse engineering of geometric models for such parts is a difficult and time consuming process fraught with difficulties due to inaccuracies, wear, deformations, and imprecision of both natural and engineered objects. The key observation is that the traditional reverse engineering and meshing pipeline may be bypassed if an object model and its boundary are described by an approximate distance field. 
Such fields may be often constructed directly from sampled data generated by 3D laser scanners or other scanning technology. Once the points on the surface of the part have been generated, a variety of methods can be used to compute an approximate distance field. In our examples the exact distance field was randomly sampled throughout the volume, and then represented by B-splines with coefficients computed using the least square method. The scan and solve approach is depicted in the flowchart in the Fig. 2.2 with operands depicted as ovals, and operations depicted as rectangles. At the outset, the geometry is scanned to produce a $2 \mathrm{D}$ or $3 \mathrm{D}$ image. Here, image refers to a regular grid of pixels or voxels for $2 \mathrm{D}$ or $3 \mathrm{D}$ geometry, respectively. The image is then segmented using image processing techniques to produce a binary image with foreground and background only. A Euclidean distance transform is then applied to compute a distance value for each image element. Samples of the distance image are taken at randomly distributed points and a set of basis functions is fit to these samples to produce an approximate distance field. The approximate distance field is then used to support meshfree simulation of stress or other physical quantities. [4].

As a demonstration of "Scan and Solve" approach, Fig 2.3 shows Scan\&Solve stress analysis performed from a SEM micrograph of fracture surface of TaC sample. The first picture in the analysis pipeline shows a SEM micrograph of fracture surface of TaC sample. The last three pictures illustrates the distributions of the components of the displacement vector and normal stress $\sigma_{y}$ (shown on the scale from 0 to $16 \mathrm{GPa}$ ). Segmentation of the SEM micrograph results in a binary image in which white color depicts material and black color corresponds to the pores. Samples of the Euclidean distance to the boundary are computed using Euclidean distance transformation. These samples are used to construct a smooth approximate distance field whose zero set describes the geometry of the boundary of a cross section. Distance fields to the fixed portions of the boundary are constructed by trimming[15]. Meshfree analysis combines distance fields, boundary conditions and basis functions to compute the displacement field and stresses. 


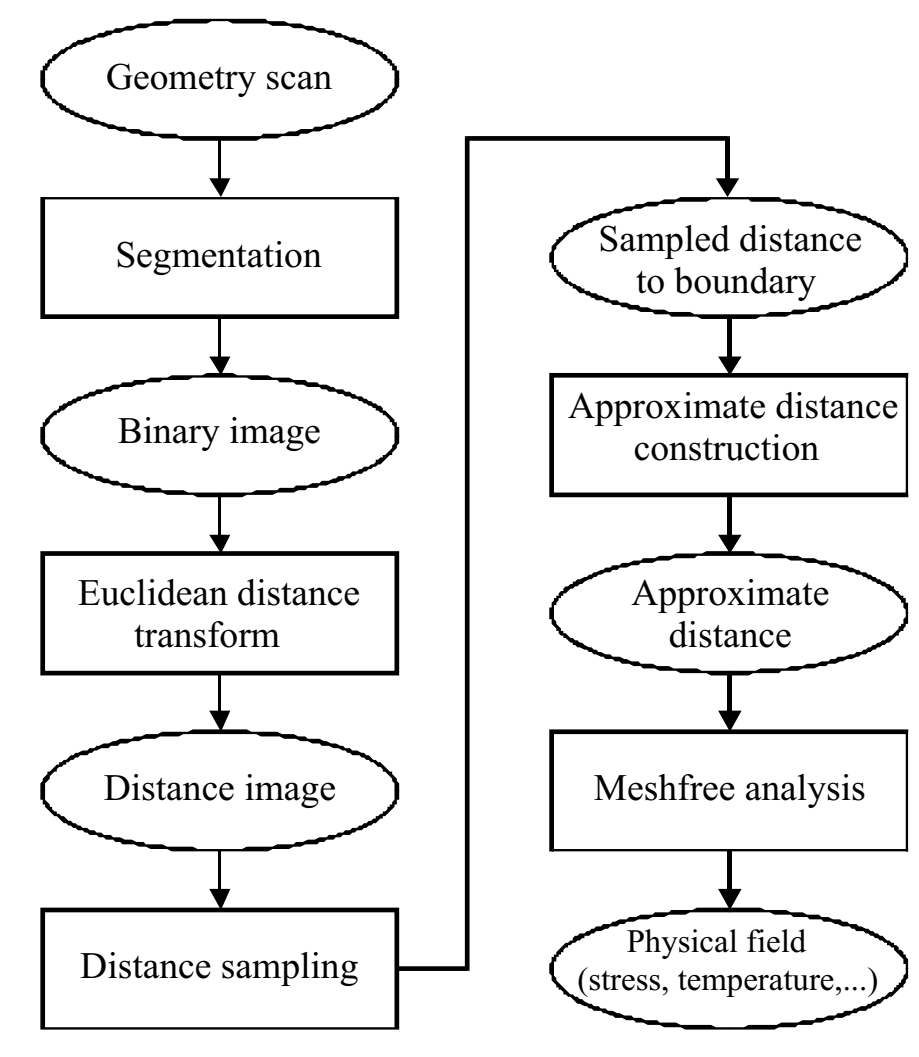

Figure 2.2: "Scan and Solve" Flowchart .

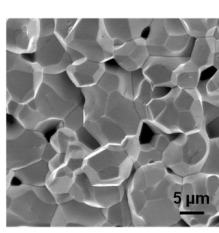

SEM micrograph

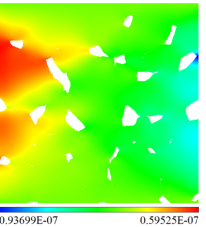

Horizontal component of the displacement

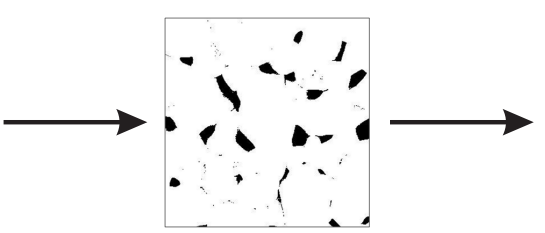

Binary image

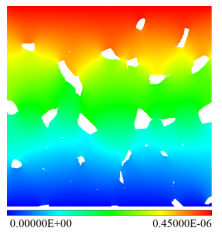

Vertical component of the displacement

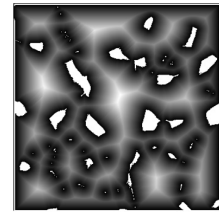

Sampled

Euclidean distance
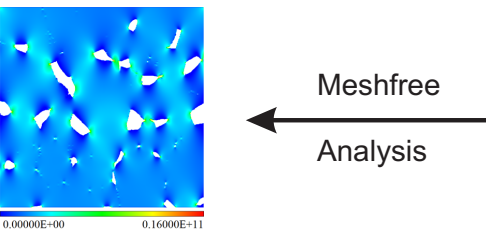

Stress $\sigma_{\mathrm{y}}$

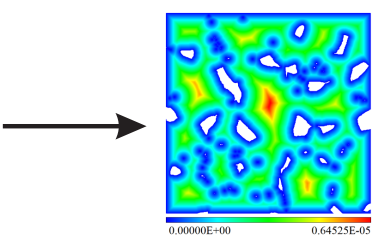

Smooth distance field

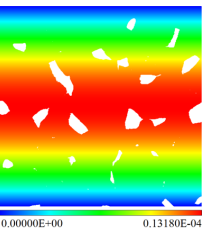

Distance field to the boundaries with prescribed displacement

Figure 2.3: Scan\&Solve stress analysis performed from a SEM micrograph of fracture surface of TaC sample. 


\section{CHAPTER 3}

\section{HOMOGENIZATION OF THERMAL PROPERTIES OF POROUS \\ CERAMIC MATERIALS USING MESHFREE METHOD WITH \\ DISTANCE FIELD}

\subsection{Asymptotic Homogenization}

In previous chapter different homogenization methods, specifically asymptotic expansion homogenization were explained and also solution structure for meshfree method were introduced. In this chapter we are going to combine both of these methods to find thermal conductivity tensor of a porous ceramic material. Applying asymptotic expansion homogenization and following the steps which was explained in chapter 2, temperature field can be expressed as follows:

$$
T(x, y)=T^{0}(x)+\varepsilon\left(T^{1}\right)(x, y)
$$

$T^{0}$ is the macroscopic or global (homogenized) temperature and $T^{1}$ is the microscopic or local temperature. The macroscopic quantities are functions of the macroscale (x) only as shown in equation (3.1). According to [10] the governing equation of heat conduction problem is described by:

$$
\int_{\Omega} k_{i j} \frac{\partial T}{\partial x_{j}} \frac{\partial T}{\partial x_{i}} d \Omega=\int_{\Omega} f \delta T d \Omega+\int_{\Gamma} h \delta T d \Gamma \forall \delta T
$$

Substituting equation (3.1) into the governing equation (3.2) and using averaging technique for a periodic function $\Psi$ in the same way with the standard homogenization procedure, the micro-macro coupled problem can be resolved into macroscopic and microscopic problems:

$$
\begin{gathered}
\lim \int_{\Omega} \psi\left(\frac{x}{\varepsilon}\right) d \Omega=\int_{\Omega} \frac{1}{|Y|} \int_{Y} \psi(y) d Y d \Omega \\
\int_{\Omega} k_{i p} \frac{\partial \phi^{j}}{\partial y_{p}} \frac{\partial \delta T^{1}}{\partial y_{i}} d Y=\int_{\Omega} k_{i j} \frac{\partial \delta T^{1}}{\partial y_{i}} d Y \forall \delta T^{1}
\end{gathered}
$$




$$
\int_{\Omega} k_{i j}^{H}\left(\frac{\partial T^{0}}{\partial x_{j}}\right)\left(\frac{\partial \delta T^{0}}{\partial x_{i}}\right) d \Omega=\int_{\Omega} f^{H} \delta T^{0} d \Omega+\int_{\Omega} h \delta T^{0} d \Gamma
$$

By solving the microscopic equation (3.4) using the periodic boundary condition, we obtain the characteristic function associated with heat conduction in the microstructure due to the mismatch of the thermal conductivities of the constituents. In equation (3.5), the homogenized thermal conductivity tensor $k_{i j}^{H}$ and the homogenized internal heat source $f^{H}$ are calculated by the integration over the whole domain and rigorously defined as follows:

$$
\begin{gathered}
k_{i j}^{H}=\frac{1}{|Y|} \int_{Y}\left(k_{i j}-k_{i p} \frac{\partial \phi^{j}}{\partial y_{p}}\right) d y \\
f^{H}=\frac{1}{|Y|} \int_{y} f d y
\end{gathered}
$$

These procedure which is used by Yasser M.Shabana and Nadotake Noda and is solved by Finite Element method. Using the same approach, we solved the problem by applying the Meshfree method.

\subsection{Representative Volume Element and Periodic Boundary Condition}

As first step, we need to define the representative volume element and the boundary condition. The RVE is usually regarded as a volume $\mathrm{V}$ of heterogeneous material that is sufficiently large to be statistically representative of the composite,or effectively include a sampling of all microstructural heterogeneities that exist in the composite. This is the general principle which is adopted, and it leads to the fact that the RVE must include a large number of the composite microheterogeneities such as: grains, inclusions, voids, fibers, etc. It must however remain small enough to be considered as a volume element of continuum mechanics [5]. Here, RVE is selected as it was shown in Fig.2.1.

After selecting the RVE, periodic boundry conditions should be applied. Usually in mathematical models and computer simulations a set of boundary condition is used to simulate the large system by modeling the small part that is far from its edge and 
since it is supposed that, most of the heterogeneous mirostructures have almost the same pattern which is repeated through the whole material, it can be called periodic boundary conditions. Figure 3.1 shows how periodic boundary condition is defined in this problem.

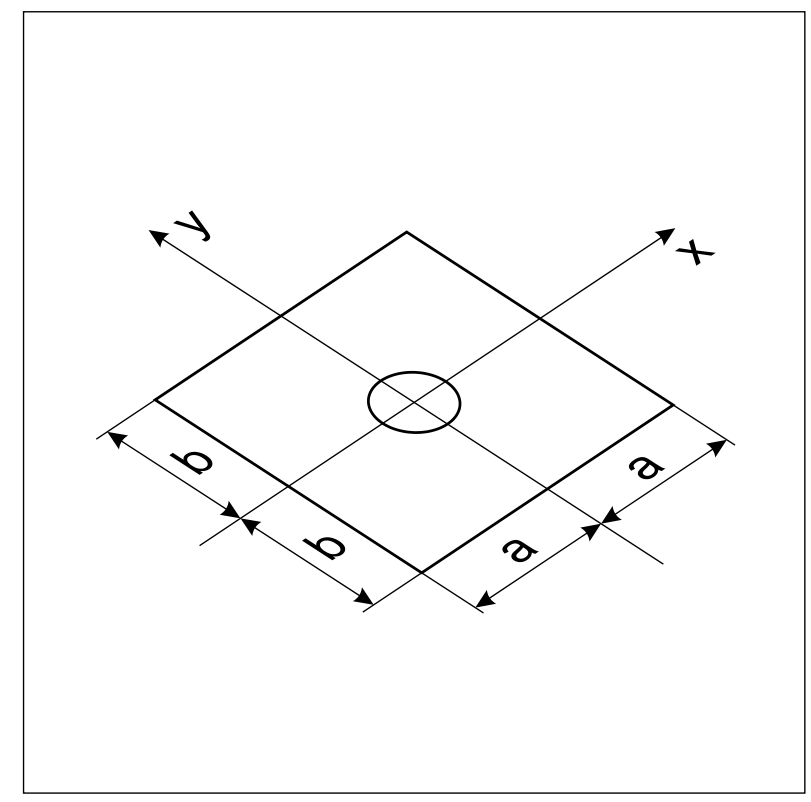

Figure 3.1: Periodic Boundary Condition

According to this figure:

$$
\begin{gathered}
\left.\phi\right|_{x=-a}=\left.\phi\right|_{x=a} \\
\left.\phi\right|_{y=-b}=\left.\phi\right|_{y=b}
\end{gathered}
$$

$\phi$ can be any function on boundaries.

\subsection{Solution Structure for Periodic Boundary Condition}

Expression(3.8) can be introduced as a solution structure for this problem. The first term provides approximation of $\phi$ inside the RVE and satisfied the homogeneous Dirichlet boundary condition on RVE's boundary and other terms are responsible for treatment of periodic boundary condition:

$$
\phi^{j}=\omega \sum_{i=1}^{n} C_{i}^{J} \chi_{i}+C_{n+1}^{J} \chi_{n+1}+C_{n+2}^{J} \chi_{n+2}+C_{n+3}^{J} \chi_{n+3}+C_{n+4}^{J} \chi_{n+4}
$$




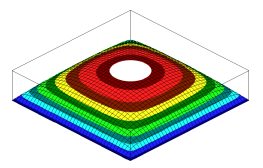

(a)

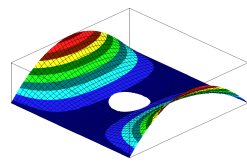

(b)

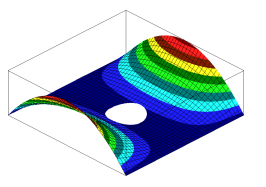

(c)

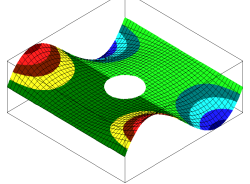

(d)

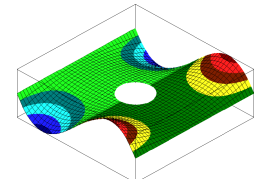

(d)

Figure 3.2: Solution structure for periodic boundary condition: (a) function $\omega$ which satisfied the homogeneous Dirichlet boundary condition; (b) shows quadratic basis function in $\mathrm{X}$ direction; (c) quadratic basis function in $\mathrm{Y}$ direction; (d) basis function of third order of polynomial in $\mathrm{X}$ direction; (e) basis function of third order of polynomial in $\mathrm{X}$ direction.

We can transfer $\omega$ inside the summation operator, so we will have:

$$
\phi^{j}=\sum_{i=1}^{n} \omega C_{i}^{J} \chi_{i}+C_{n+1}^{J} \chi_{n+1}+C_{n+2}^{J} \chi_{n+2}+C_{n+3}^{J} \chi_{n+3}+C_{n+4}^{J} \chi_{n+4}
$$

$\xi$ is defined in this way:

$$
\xi= \begin{cases}\omega \chi_{i} & i=1, \ldots n \\ \chi & i=n+1, \ldots . n+4\end{cases}
$$

then $\xi$ is substituted in equation (3.9), so final shape function will be expressed as:

$$
\phi^{j}=\sum_{i=1}^{n+4} C_{i}^{J} \xi_{i}
$$

\subsection{Approach}

So, up to here, a shape function is produced which satisfied both homogenous Dirichlet and periodic boundary conditions, now we could substitute this shape function into the microscopic heat conduction problem equation. Therefore, equation (3.4), will turn to:

$$
\int_{\Omega} k_{i p} \frac{\partial \phi^{j}}{\partial y_{p}} \frac{\partial \xi_{k}}{\partial y_{i}} d \Omega=\int_{\Omega} k_{i j} \frac{\partial \xi_{k}}{\partial y_{i}} d \Omega
$$


This equation could be considered as a system of algebraic equation like $[A][C]=$ $[B]$ in which:

$$
\begin{gathered}
a_{k m}=\int_{\Omega} k_{i p} \frac{\partial \xi_{m}}{\partial y_{p}} \frac{\partial \xi_{k}}{\partial y_{i}} d \Omega \\
b_{k}=\int_{\Omega} k_{i j} \frac{\partial \xi_{k}}{\partial y_{i}} d \Omega
\end{gathered}
$$

Solving this algebraic equations system, will result in finding $C_{i}^{J}$ coefficients which can be substituted in shape function equation and find the $\phi^{j}$ from equation (3.11). Finally using the calculated shape function in equation (3.6), we will get the homogenized thermal conductivity. The homogenization procedure is summarized in Fig3.3.

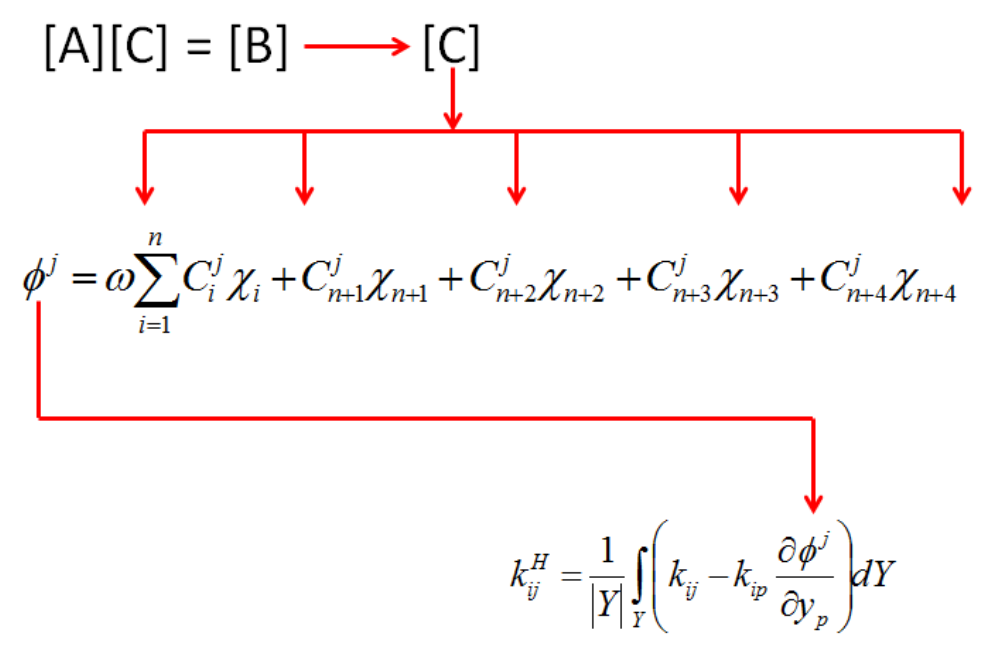

Figure 3.3: Summary of homogenization procedure

\subsection{Benchmarks}

Since, there are no related experimental results available in this field in terms of thermal conductivity tensor, for checking the consistency of the proposed method, we 
set up some numerical experiments to demonstrate this momentous. The goal, initial data and results are explained precisely in each experiment.

\subsubsection{Test 1 : Homogeneous material with no porosity}

The goal of this experiment is to demonstrate that the proposed approach can reconstruct the originally specified material properties in the absence of porosity. We consider a rectangular as with this initial data: $\mathrm{k}=\left[\begin{array}{ll}1 & 0 \\ 0 & 1\end{array}\right], a=b=1$.

In this case, since we do not have any heterogeneity, so we should expect to get the same result as the homogenous thermal conductivity. performing homogenization for this problem, we get $k^{H}=\left[\begin{array}{ll}1 & 0 \\ 0 & 1\end{array}\right]$.

\subsubsection{Test 2: Material with rectangular pattern of the circular holes}

The goal of this experiment is to demonstrate that selection of different RVE's for one material, will not affect the homogenized thermal conductivity tensor. If we consider a domain with rectangular pattern of circular holes, Fig 3.4, there are many possibilities of selecting different RVE's and in between there are two possibilities for rectangular RVE's, one is a square with a hole in center and another one is a square with quadrant in corners. The initial data for both cases are: $\mathrm{k}=\left[\begin{array}{ll}1 & 0 \\ 0 & 1\end{array}\right]$, $R=0.25, a=b=1$.

Fig 3.4, shows the RVE's and also shape functions of both cases $\phi^{j}$, which are generated regarding to the selected geometry and distance function which were explained in previous chapter.

Although we have two different RVE's here, but since we are dealing with one material, we expected to get the same thermal conductivity for each case. The obtained result for first RVE which was a square with a hole in center is: $k^{H}=\left[\begin{array}{cc}0.906622 & 0 \\ \sum 0 & 0.906622\end{array}\right]$, and result for second RVE which was a square with quadrant in corners is: $k^{H}=$ $\left[\begin{array}{cc}0.908391 & 0 \\ 0 & 0.908391\end{array}\right]$. 


\begin{tabular}{|l|l|l|l|l|}
\hline$\circ$ & 0 & 0 & 0 & 0 \\
\hline 0 & 0 & 0 & 0 & 0 \\
\hline 0 & 0 & 0 & 0 & 0 \\
\hline 0 & 0 & $\circ$ & 0 & 0 \\
\hline 0 & 0 & 0 & 0 & 0 \\
\hline
\end{tabular}

(a)

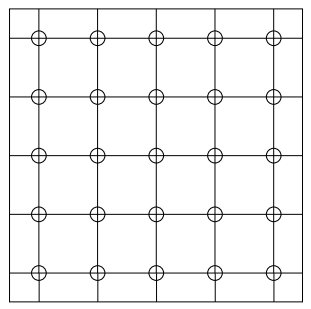

(e)

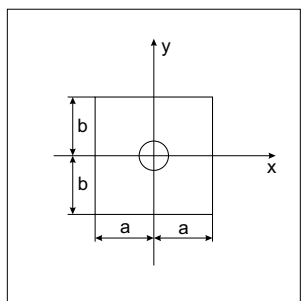

(b)

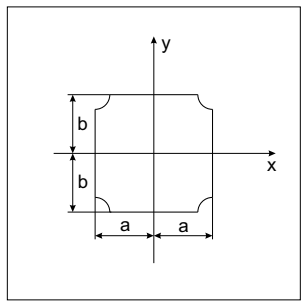

(f)

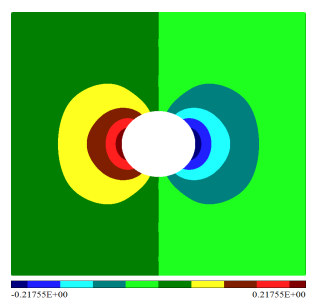

(c)

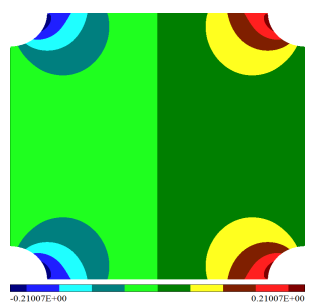

(g)

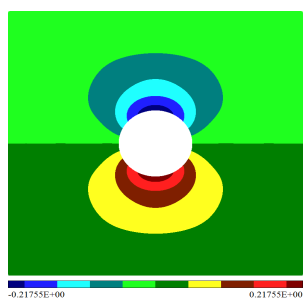

(d)

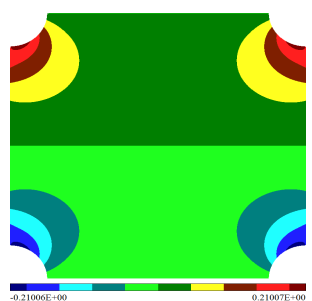

(h)

Figure 3.4: Two different RVE's and their shape functions for a material with rectangular pattern of the circular holes

As it can be seen, the results are in good agreement. The very close results, also, indicate the consistency of the proposed method. Furthermore, in order to make sure that the results are reliable, we did convergence study and check the convergence of results by increasing the degree of polynomials of basis function. Convergence graph can be seen in Fig 3.5.

\subsubsection{Test 3: Applying standard simulation software approach (SSSA)}

As a matter of fact, not only Asymptotic Expansion Homogenization (AEH) could be applied to find homogenized thermal conductivity of porous ceramic materials, but also other approaches like those which are used by standard simulation software packages could be used. The goal of this experiment is to solve the problem with other approach rather than $\mathrm{AEH}$ and compare the results. To do so, first we are going to explain how standard simulation software approach works, then using this approach, solve the problem both by ANSYS and Meshfree method.

The heat flux for nonhomogeneous material in two dimension could be written as: $q=$ $-\left[\begin{array}{ll}k_{11} & k_{12} \\ k_{21} & k_{22}\end{array}\right]\left[\begin{array}{l}T_{x} \\ T_{y}\end{array}\right]$ 


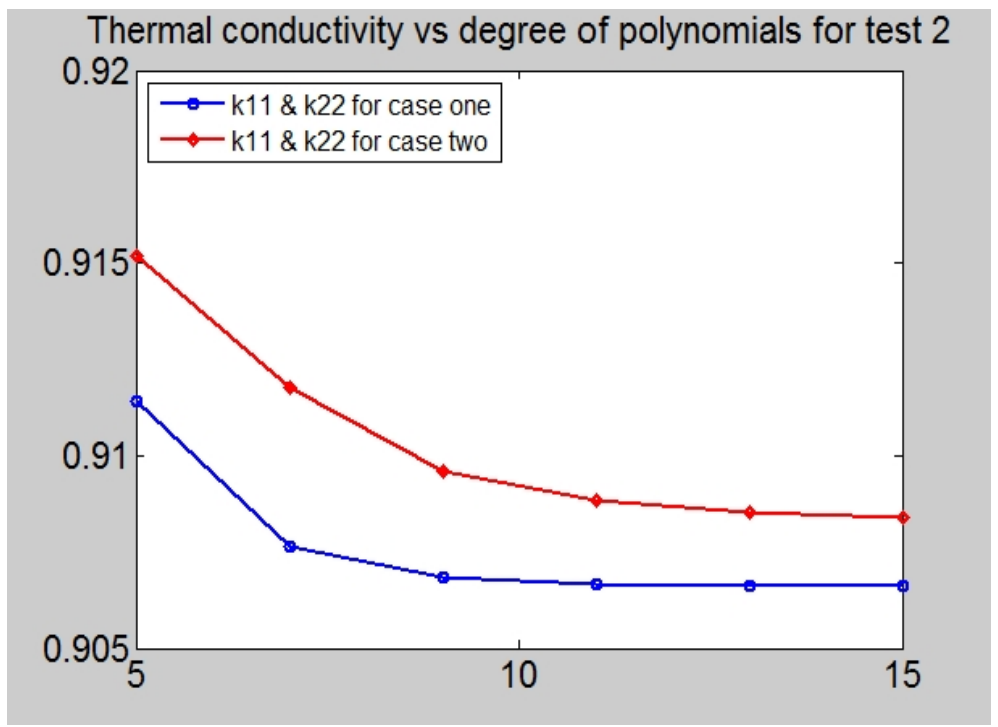

Figure 3.5: Convergence study for test 2: case one is a square with a hole in center and case two is a square with quadrant in corners

So, we have:

$$
q_{x}=-\left(k_{11} T_{x}-k_{12} T_{y}\right)
$$

and

$$
q_{y}=-\left(k_{21} T_{x}-k_{22} T_{y}\right)
$$

which $T_{x}$ and $T_{y}$ are partial derivatives of temperature. Therefore, for performing homogenization we have to divide the problem in two parts, first applying the temperature gradient in $X$ direction and found $\overline{q_{x}}$ and $\overline{q_{y}}$ with applying these boundary condition:

$$
\left.T\right|_{x=0}=0
$$

and

$$
\left.T\right|_{x=2 a}=1
$$

and assume adiabatic condition at $Y$ direction. In this case, $T_{y}$ become zero and we can 
find $k_{11}$ and $k_{21}$ from these equations:

$$
\begin{gathered}
q_{x}=-k_{11} T_{x} \\
q_{y}=-k_{21} T_{x}
\end{gathered}
$$

In next step, the problem is solved by applying the temperature gradient in $Y$ direction with these boundary conditions:

$$
\left.T\right|_{y=0}=0
$$

and

$$
\left.T\right|_{y=2 b}=1
$$

and assume adiabatic condition at $X$ direction. In the same way, $T_{x}$ become zero, so we can find $k_{12}$ and $k_{22}$ from these equations:

$$
\begin{aligned}
& q_{x}=-k_{12} T_{y} \\
& q_{y}=-k_{22} T_{y}
\end{aligned}
$$

This is the general approach which is used by standard simulation software packages. Fig.3.6 also shows the schematic of the approach.
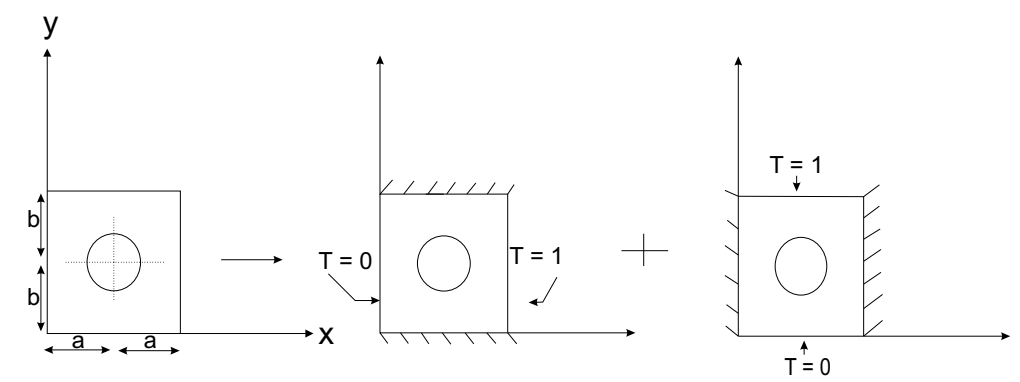

Figure 3.6: General schematic of standard simulation packages Approach 
Now, we are going to solve our problem in ANSYS. In order to have comparable results, we choose the same RVE such as the previous test. A rectangle with a hole in center ,Fig 3.4.b, and these initial data:

$\mathrm{k}=\left[\begin{array}{ll}1 & 0 \\ 0 & 1\end{array}\right], R=0.25, a=b=1$. Then, the selected geometry was meshed with the ANSYS standard options. As it was explained, we applied temperature gradient in two steps in $\mathrm{X}$ and $\mathrm{Y}$ direction.

\begin{tabular}{|c|c|c|c|}
\hline \multicolumn{3}{|c|}{ Details of "User Defined Result" } & प \\
\hline \multirow[t]{3}{*}{$\square$} & \multicolumn{3}{|l|}{ Scope } \\
\hline & Scoping Method & \multicolumn{2}{|l|}{ Geometry Selection } \\
\hline & Geometry & \multicolumn{2}{|l|}{ All Bodies } \\
\hline \multirow[t]{10}{*}{$\square$} & \multicolumn{3}{|l|}{ Definition } \\
\hline & Type & \multicolumn{2}{|l|}{ User Defined Result } \\
\hline & Expression & \multicolumn{2}{|l|}{$=\mathrm{TFY}$} \\
\hline & Input Unit System & \multicolumn{2}{|l|}{ Metric $(\mathrm{m}, \mathrm{kg}, \mathrm{N}, \mathrm{s}, \mathrm{V}, \mathrm{A})$} \\
\hline & \multicolumn{3}{|l|}{ Output Unit } \\
\hline & By & \multicolumn{2}{|l|}{ Time } \\
\hline & Display Time & \multicolumn{2}{|l|}{ Last } \\
\hline & Coordinate System & \multicolumn{2}{|l|}{ Global Coordinate System } \\
\hline & Calculate Time History & \multicolumn{2}{|l|}{ Yes } \\
\hline & Identifier & & \\
\hline \multirow[t]{2}{*}{$\square$} & \multicolumn{3}{|c|}{ Integration Point Results } \\
\hline & Display Option & Averaged & $\nabla$ \\
\hline \multirow[t]{3}{*}{$\square$} & Results & Unaveraged & $\Delta$ \\
\hline & Minimum & \multirow{3}{*}{$\begin{array}{l}\text { Nodal Difference } \\
\text { Nodal Fraction } \\
\text { Elemental Difference } \\
\text { Elemental Fraction }\end{array}$} & \\
\hline & $\square$ Maximum & & $=$ \\
\hline 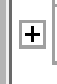 & Information & & $\nabla$ \\
\hline
\end{tabular}

Figure 3.7: ANSYS User Define Result Window

After solving the problem, the contour of temperature distribution was shown as in Fig 3.8, but temperature distribution is not enough for us, because we need to find heat flux in $\mathrm{X}$ and $\mathrm{Y}$ direction according to equation 3.15 and 3.16. As a result, we tried to find a way that enables us to manage the ANSYS results. "User Defined Result" button is used for this purpose. Clicking on this button, will open a new window such as 


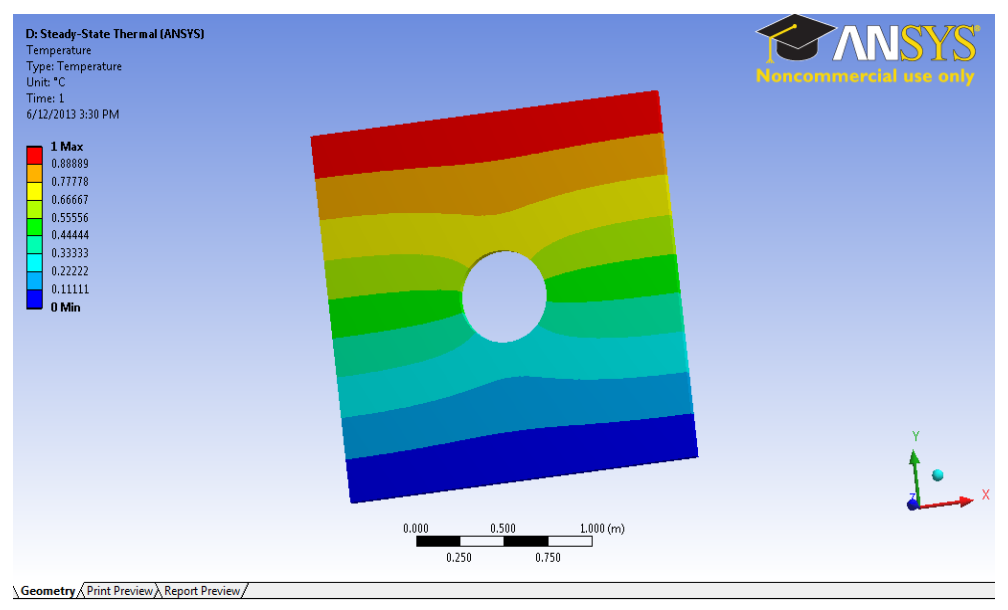

Figure 3.8: Temperature Distribution in Y direction

Fig 3.7, which gave us this ability to control showing the results in more details. Using this option, we can get the heat flux distribution in $\mathrm{X}$ and $\mathrm{Y}$ direction.

For finding the homogenized thermal conductivity tensor, the heat flux should be integrated over the domain such as below:

$\bar{q}_{x}=\int q_{x} d x / A$ and $\bar{q}_{y}=\int q_{y} d y / A$. But since ANSYS did not support integrating the results in this way, so we had to export the information of each element into the Excel file and averaged them discretely to get the averaged amount of heat flux according to the following formulation:

$\bar{q}_{x}=\left(\sum_{i=1}^{n} q_{x i}\right) / n$. where "n" is the number of the elements. We solved the problem for both cases and found the averaged heat flux in $\mathrm{X}$ and $\mathrm{Y}$ directions and applied equations (3.17), (3.18), (3.19) and (3.20 to find the thermal conductivity tensor. Here are the results of this test:

$k^{H}=\left[\begin{array}{cc}0.9523 & 0 \\ 0 & 0.9541\end{array}\right]$ and at last, using the same approach, we solve the problem with Meshfree method and get the following result as homogenized thermal conductivity tensor: $k^{H}=\left[\begin{array}{cc}0.908626 & 0 \\ 0 & 0.908393\end{array}\right]$

First, comparing this result with the previous ones shows the $4.8 \%$ differences between result of meshfree method and ANSYS as one of the most powerful finite element packages. This is because of the ANSYS limitations in performing the integration over the physical domain for homogenization purposes and once more, pointed the weakness of 
finite element based packages for finding the accurate physical properties of material. Second, we can see that this result are almost identical with the results in test 2 and show that, although two different approaches were used in these two tests, but we got the same result for one macrostructure.

\subsection{More Complicated Patterns}

Since porous ceramic materials have both isotropic and anisotropic microstructure, we try to find homogenized thermal conductivity for both cases by testing different RVE's. So, in the following, results for more complicated pattern is presented and it is tried to accommodate anisotropic RVE's as well. Periodic boundary condition is applied in all the cases. Each example is solved in meshfree (AEH), (SSSA) and ANSYS and results brought for comparison. In addition, convergence study for each case has been done and the graphs are given.

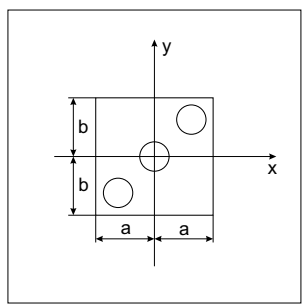

(a)

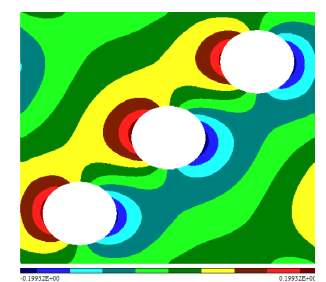

(b)

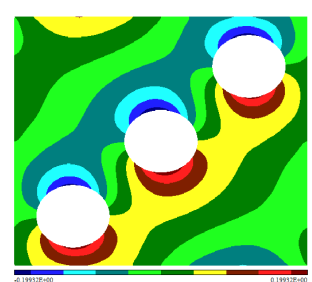

(c)

Figure 3.9: RVE and shape function for example 1

\subsubsection{Example 1: square with three diagonal circle}

The initial data for this case is : $\mathrm{k}=\left[\begin{array}{ll}1 & 0 \\ 0 & 1\end{array}\right], R=0.25, a=b=1$. Meshfree AEH result for example 1 is: $k^{H}=\left[\begin{array}{ll}0.743341 & 0.021001 \\ 0.021001 & 0.743341\end{array}\right]$. As result shows, $k_{11}$ and $k_{22}$ are equal so there is equal thermal conductivity in $\mathrm{X}$ and $\mathrm{Y}$ directions. This can be related to the symmetry of the geometry of this RVE and the equal amount of material distribution in each direction. The convergence graph is shown in Fig 3.10. Meshfree $(\mathrm{SSSA})$ result are for this case is: $k^{H}=\left[\begin{array}{ll}0.756382 & 0.018629 \\ 0.018521 & 0.756014\end{array}\right]$. Ansys result for this 
geometry is: $k^{H}=\left[\begin{array}{ll}0.857957 & 0.057042 \\ 0.055536 & 0.865689\end{array}\right]$. Comparison between the results show that

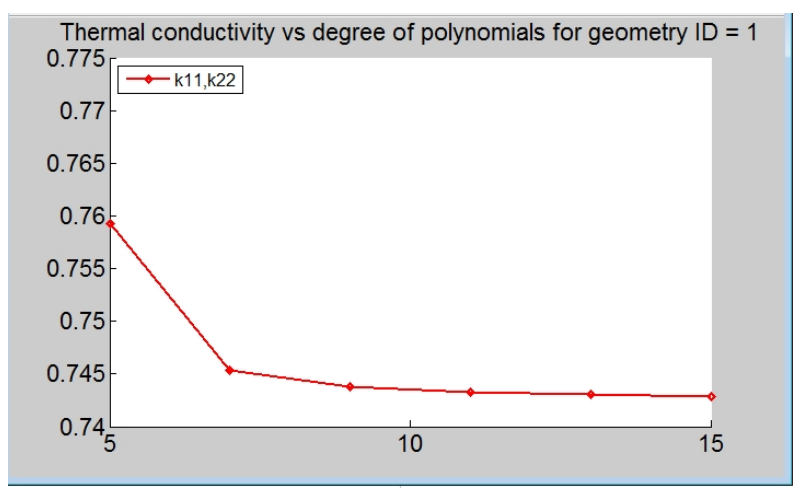

(a)

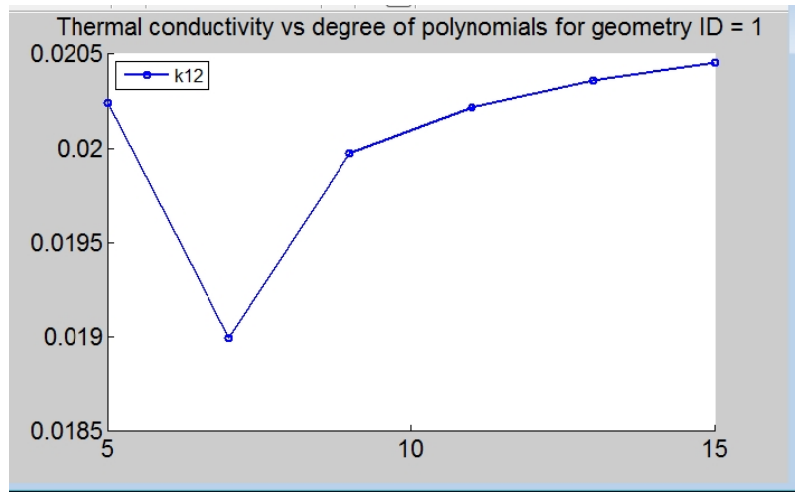

(b)

Figure 3.10: Convergence graph for example 1

unlike ANSYS result, the meshfree results for both AEH and SSSA are identical. This is because of ANSYS limitation in performing integration over domain.

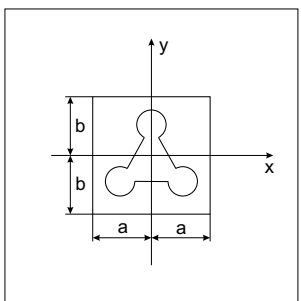

(a)

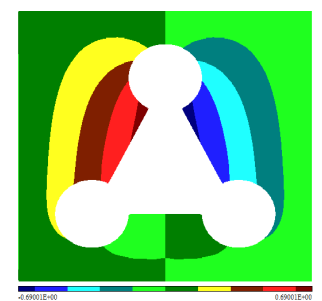

(b)

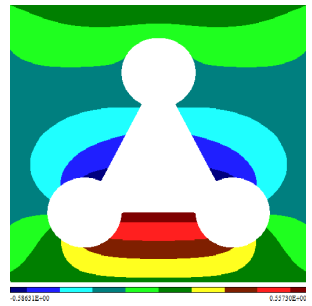

(c)

Figure 3.11: RVE and shape functions for example 2

\subsubsection{Example 2: square with triangle inside and holes}

The initial data for this case is : $\mathrm{k}=\left[\begin{array}{ll}1 & 0 \\ 0 & 1\end{array}\right], R=0.25, a=b=1$. Meshfree AEH result for Example 2 is: $k^{H}=\left[\begin{array}{cc}0.428356 & 0 \\ 0 & 0.478928\end{array}\right]$. As it is obvious here, since we have smaller porosity coefficient than the previous example, the thermal conductivity become less too. Also, in this example, the thermal conductivity in $\mathrm{X}$ and $\mathrm{Y}$ direction are not exactly the same but close to each other and the off diagonal components are zero which means that $k_{12}$ is zero. This situation shows an orthotropic behavior. The conver- 
gence graph is shown in Fig 3.12. Also the SSSA result is: $k^{H}=\left[\begin{array}{cc}0.430363 & 0 \\ 0 & 0.483600\end{array}\right]$. The ANSYS result for this RVE is: $k^{H}=\left[\begin{array}{ll}0.557629 & 0.001405 \\ 0.005423 & 0.631368\end{array}\right]$. Comparing these three results, show that the meshfree results for both AEH and SSSA are almost the same, while ANSYS result is much different and it could be because of the same reason which was explained before.

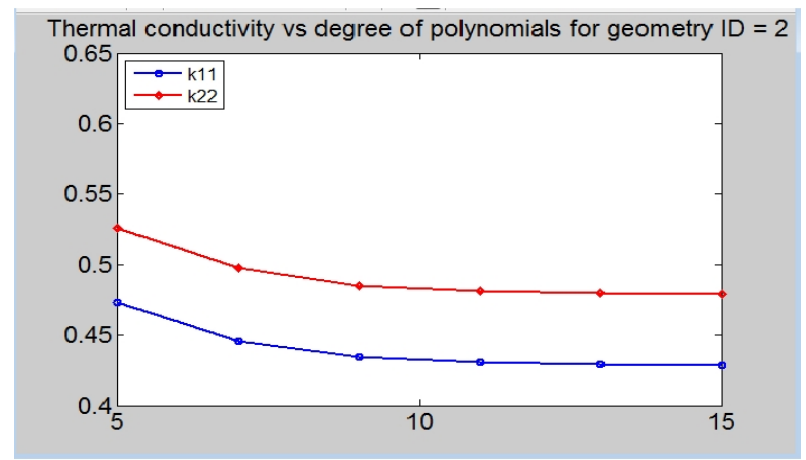

Figure 3.12: Convergence graph for example 2

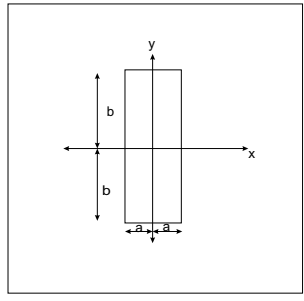

(a)

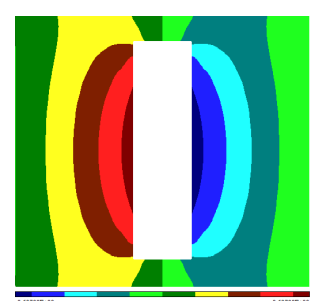

(b)

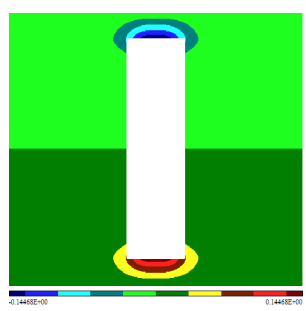

(c)

Figure 3.13: RVE and shape function for example 3

\subsubsection{Example 3: square with rectangle inside}

The initial data for this case is : $\mathrm{k}=\left[\begin{array}{ll}1 & 0 \\ 0 & 1\end{array}\right], a=0.2, b=0.8$. Meshfree AEH result for example 3 is: $k^{H}=\left[\begin{array}{cc}0.401291 & 0 \\ 0 & 0.815216\end{array}\right]$. In this example, again we have larger amount of material in $\mathrm{X}$ direction than $\mathrm{Y}$, so the $k_{11}$ is lager than $k_{22}$ and since we have different amounts in $\mathrm{X}$ and $\mathrm{Y}$ direction, therefore this material can be considered anisotropic and also since the off diagonal terms are zero, it is 
orthotropic as well. The convergence graph is shown in Fig 3.14. Meshfree SSSA result is: $k^{H}=\left[\begin{array}{cc}0.402193 & 0 \\ 0 & 0.817064\end{array}\right]$. The Ansys result is: $k^{H}=\left[\begin{array}{cc}0.473586 & 0.003258 \\ 0.00031 & 0.961689\end{array}\right]$. Close results can be seen in both meshfree approaches and rough result for ANSYS. Results also showed the anisotropy of homogenized thermal conductivity very well. It is one of the privilege of using meshfree method homogenization because other methods like "role of mixture" which frequently used in this kind of problem does not show the anisotropy.

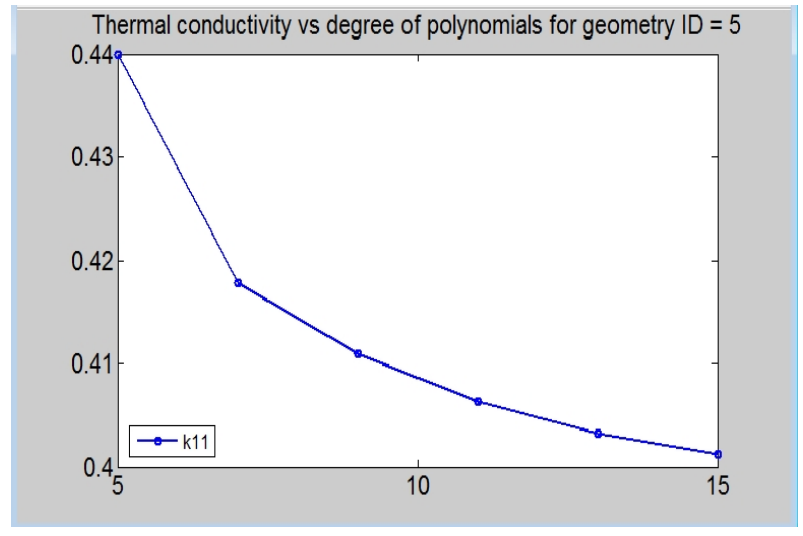

(a)

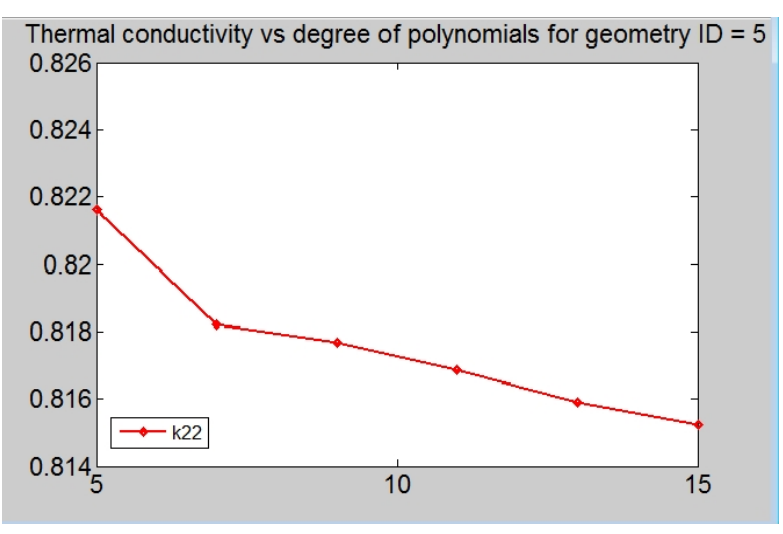

(b)

Figure 3.14: Convergence graph for example 3
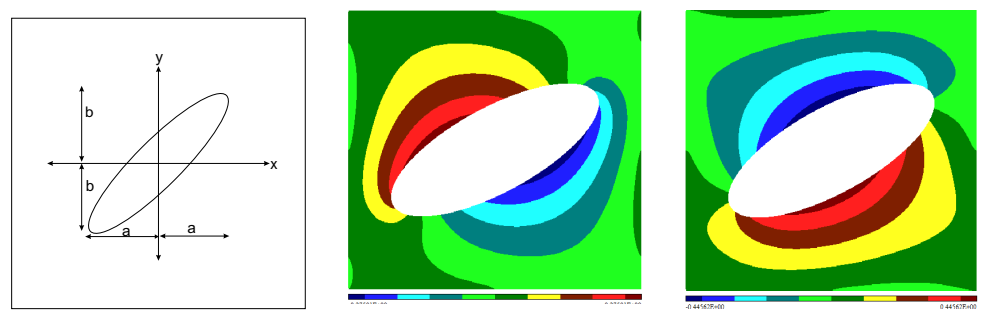

Figure 3.15: Example 4

\subsubsection{Example 4: square with ellipse inside}

The initial data for this case is $: \mathrm{k}=\left[\begin{array}{ll}1 & 0 \\ 0 & 1\end{array}\right], a=0.3, b=0.8$.

Meshfree method for example $4: k^{H}=\left[\begin{array}{ll}0.709890 & 0.109712 \\ 0.109712 & 0.561575\end{array}\right]$. Again, the role of orientation is obvious in this RVE and it has different amounts of thermal conductivity in 
each direction, so it is anisotropic. This RVE is comparable to RVE in example 3, the difference in the slope of the pore result in changing the off-diagonal components from 0 to 0.109712. The convergence graph is shown in fig 3.16. Meshfree SSSA result is: $k^{H}=\left[\begin{array}{cc}0.71228 & 0.111013 \\ 0.110487 & 0.563759\end{array}\right]$.

The Ansys result is: $k^{H}=\left[\begin{array}{cc}0.837510 & 0.007002 \\ 0.055770 & 0.72824\end{array}\right]$.

In this example, also, ANSYS result is different from two meshfree approaches.
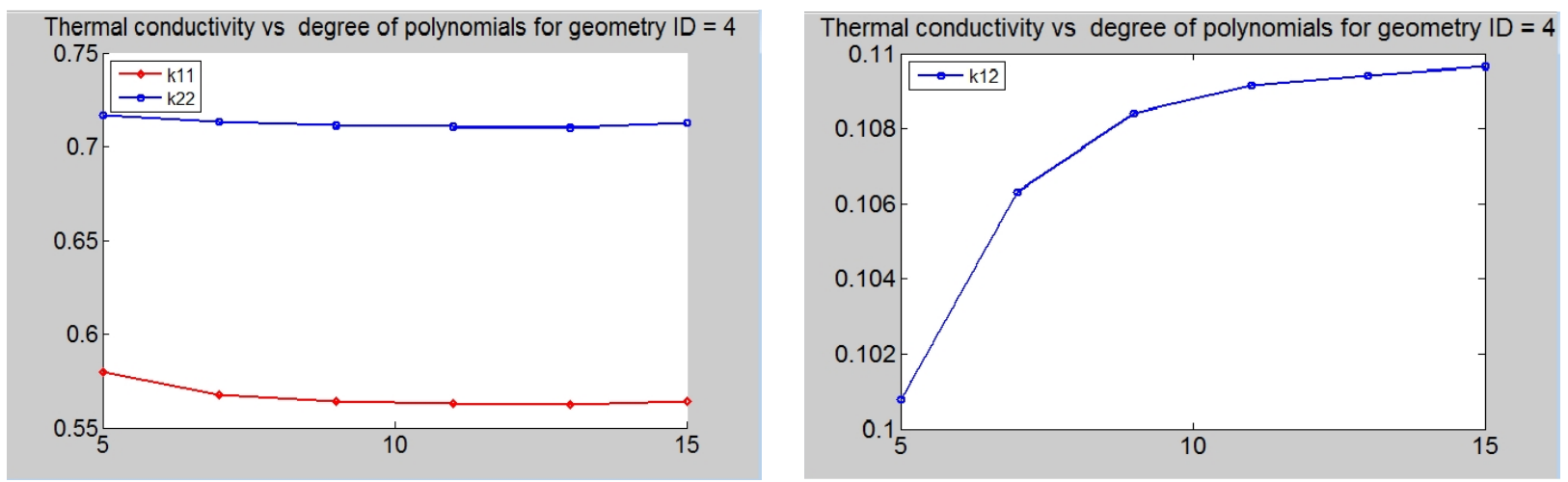

Figure 3.16: Convergence graph for example 4
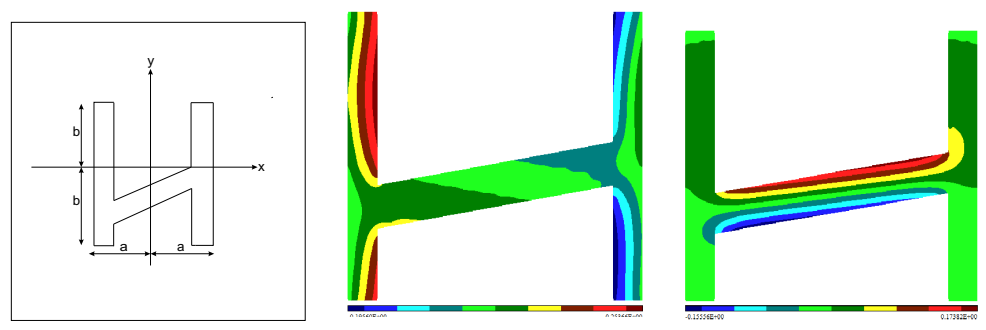

Figure 3.17: RVE and shape function for example 6

\subsubsection{Example 6: H shape}

The initial data for this case is : $\mathrm{k}=\left[\begin{array}{ll}1 & 0 \\ 0 & 1\end{array}\right], a=0.3, b=0.8$. Meshfree method for example 6 is: $k^{H}=\left[\begin{array}{ll}0.168535 & 0.020295 \\ 0.020302 & 0.211362\end{array}\right]$. An inclined beam can be seen in the geometry of this RVE that make the material to show anisotropic behavior. As results show, not only the $k_{11}$ and $k_{22}$ are different, also the off diagonal 
components are not zero and it describes the situations when properties depend on the direction. The convergence graph is shown in Fig 3.18. Meshfree SSSA result for this example is: $k^{H}=\left[\begin{array}{cc}0.160483 & 0.021491 \\ 0.0202466 & 0.211704\end{array}\right]$. The Ansys result for example 5 is $: k^{H}=\left[\begin{array}{cc}0.432033 & 0.0581818 \\ 0.0353719 & 0.7049917\end{array}\right]$. In all of these example, the meshfree AEH and SSSA results were almost identical, while the ANSYS results were different. Although all the results were approximated, but since two of them were more close to each other, therefore, we can conclude that those two, are more accurate. It was also concluded that the rough results of ANSYS is because of the limitation of this simulation software package in performing integration over the domain.
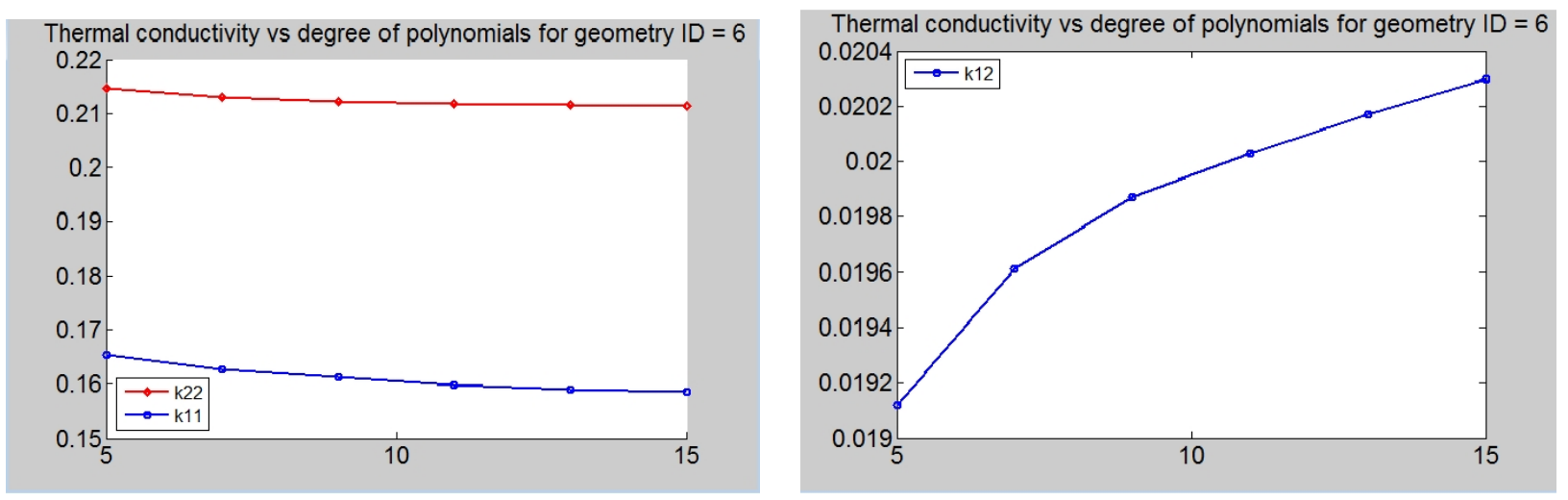

Figure 3.18: Convergence graph for example 5

\subsection{Realistic Microstructure Obtained From the Micrograph}

In this part, we are going to accomplish our commitment in the research and apply our method on realistic geometry of microstructure. In this regard, we got a microstructure which is obtained from the micrograph. We used the TaC 1800 micrograph and applied our method to find it's homogenized thermal properties.

As it is shown in Fig 3.19, we need to convert the gray picture to binary (black and white) picture. It can be done by the help of software like Coral Photo Paint, Irfan View and Paint. Since Meshfree method works with distance functions in constructing the domain, we need to have the exact information of each point and their distance to 
the boundary. So, here, it is needed to convert the binary image to some kind of image that have the facility to give us the distance information. We used the "Skeleton " software for this mean. The obtained data from the "Skeleton "software have been used in the code, and rest of the work is similar to previous examples. Finally we got homogenized thermal conductivity tensor as:

$$
K^{H}=\left[\begin{array}{cc}
0.430363 & 0 \\
0 & 0.483600
\end{array}\right] .
$$

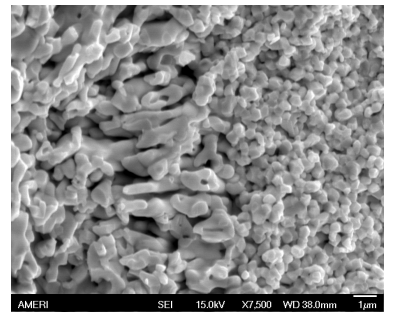

SEM micrograph

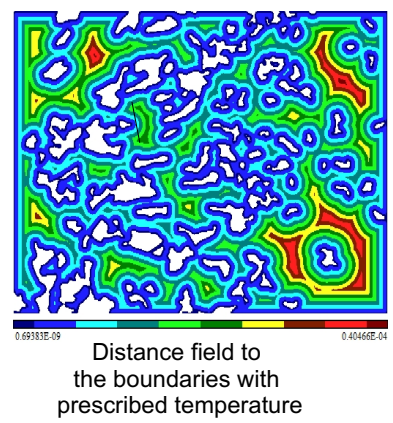

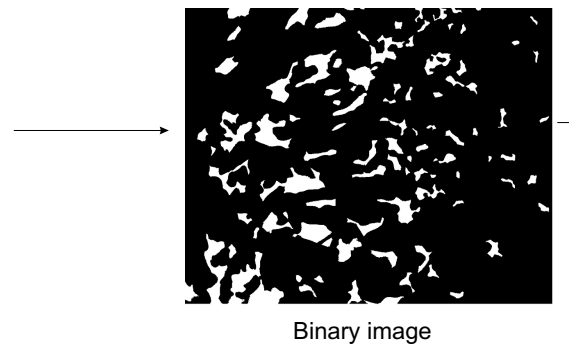

Binary image

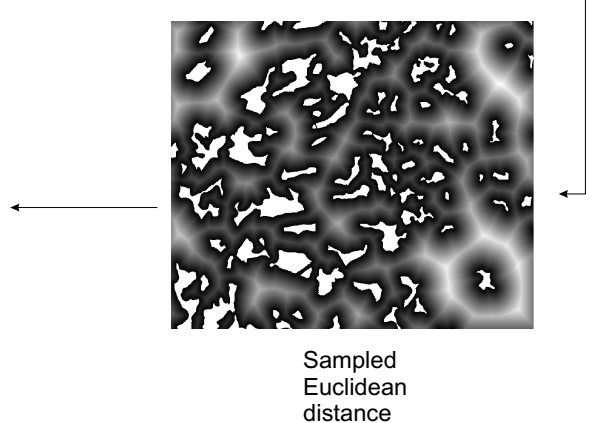

Figure 3.19: Scan\&Solve thermal analysis performed from a SEM micrograph of surface of $\mathrm{TaC}$ sample. 


\section{CHAPTER 4}

\section{INVESTIGATING THE DEPENDENCE OF THE ANISOTROPY OF THERMAL CONDUCTIVITY ON GEOMETRIC PROPERTIES}

In this chapter, series of targeted experiments are done to investigate the dependence of the anisotropy of thermal conductivity of porous ceramic materials on geometric properties of their microstructure. There are some important geometric parameters of microstructure that can affect the homogenized thermal conductivity. Porosity coefficient, pores orientation and also the density of pores are some of these important factors. Therefore, we are going to set up an experiment to study the rate of changes of thermal conductivity regarding these parameters.

To do so, a square will be used as an RVE with a rectangle in it. This rectangle which later will be called "cloud of porosity" has three specifications. First, it includes selected number of circles or ellipses which play the role of porosity in the experiments. Second, it will rotate 180 degree through each experiment. This rotation will help to investigate role of orientation of the porosity structure. Third the pores will be distributed randomly within the rectangle.

In order to investigate the effect of porosity coefficient, we will change the parameters such as geometry of pores which could be circle or ellipse, size and number of them and also dimensions of cloud of porosity . We can run several experiments with different porosity coefficients and show the results on the graphs. Also, for checking the role of orientation, we are going to rotate the cloud of porosity and find the related thermal conductivity in every selected angle. In this way we could depict the variations of the principle geometric axes with respect to the orthotropic axes and see the correlation between them.

Before starting to explain the experiments, it will be helpful to define what the principal geometric and anisotropy axes are.

In this research we are considering two dimensional problems, so matrix notation for moment of inertia will be: $\left[\begin{array}{cc}I x x & I x y \\ I x y & I y y\end{array}\right]$ 
and we could find the position of principal axes of geometry, using this equation:

$$
\alpha_{P}=\frac{\arctan \frac{2 \times I_{x y}}{I_{y y}-I_{x x}}}{2}
$$

Axes of anisotropy for the thermal conductivity will be defined in the same way, using thermal conductivity tensor components:

$$
\alpha_{O}=\frac{\arctan \frac{2 \times k_{12 h}}{k_{11 h}-k_{22 h}}}{2}
$$

As it was said before, we could investigate the affect of orientation of pores to the anisotropy of homogenized thermal conductivity, by comparing these two angles.

\subsection{Experiment A:}

In this experiment the geometry of the pores is selected to be ellipses with these dimensions: $\mathrm{a}=0.04, \mathrm{~b}=0.02$. Also the dimension of the cloud of porosity is fixed to $(0.8 \times 0.15)$. Keeping these parameters untouched, we run the code for three different number of ellipses, 10, 30, 50 separately and plot the results as following. The porosity coefficient for these three case is: $0.006,0.018,0.028$.

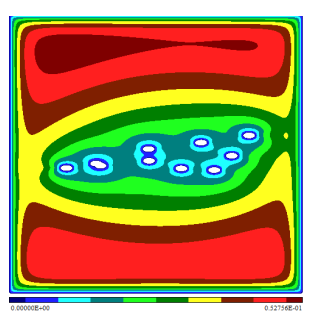

(a)

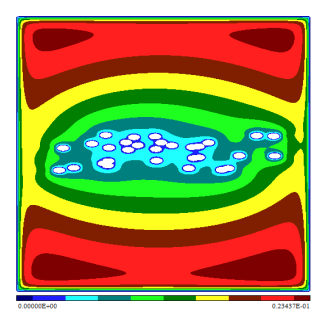

(b)

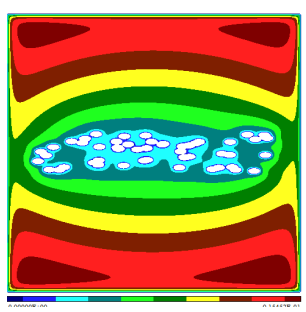

(c)

Figure 4.1: Cloud of porosity for experiment A, geometry of pores are ellipses $(\mathrm{a}=0.04$, $\mathrm{b}=0.02)$ and cloud of porosity dimension is: $(0.8 \times 0.15)$

\subsubsection{Discussion}

Figures 4.2 and 4.3 show that thermal conductivity will decrease when porosity coefficient increases. Higher porosity coefficient means smaller amount of material which will result in smaller amount of thermal conductivity. Also, as it can be seen, the changes 


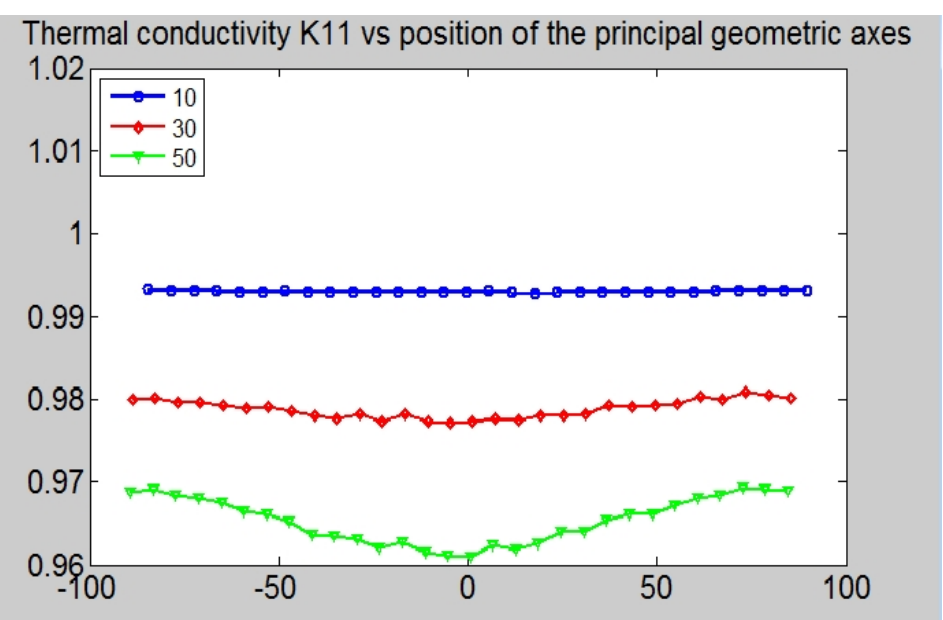

Figure 4.2: $k_{11}$ for Experiment A, geometry of pores are ellipses $(\mathrm{a}=0.04, \mathrm{~b}=0.02)$ and cloud of porosity dimension is: $(0.8 \times 0.15)$

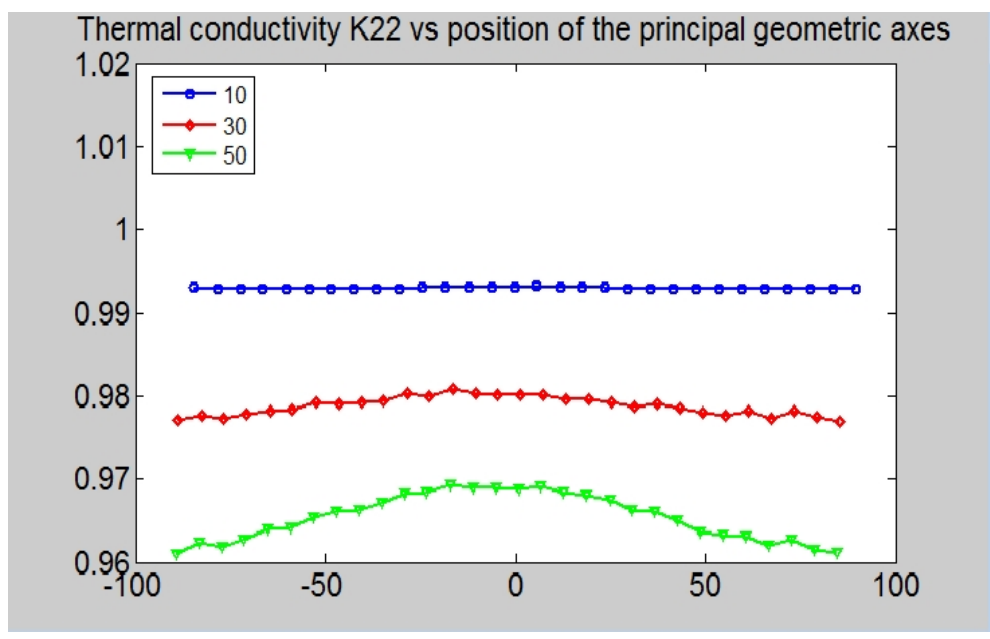

Figure 4.3: $k_{22}$ for Experiment A, geometry of pores are ellipses $(\mathrm{a}=0.04, \mathrm{~b}=0.02)$ and cloud of porosity dimension is: $(0.8 \times 0.15)$ 


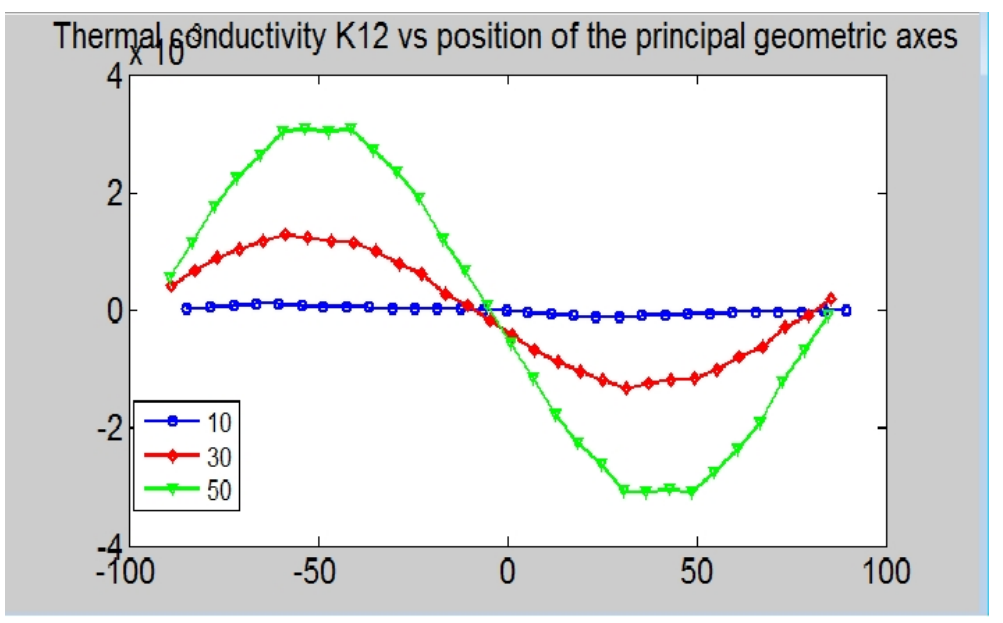

Figure 4.4: $k_{12}$ for Experiment A, geometry of pores are ellipses $(\mathrm{a}=0.04, \mathrm{~b}=0.02)$ and cloud of porosity dimension is: $(0.8 \times 0.15)$

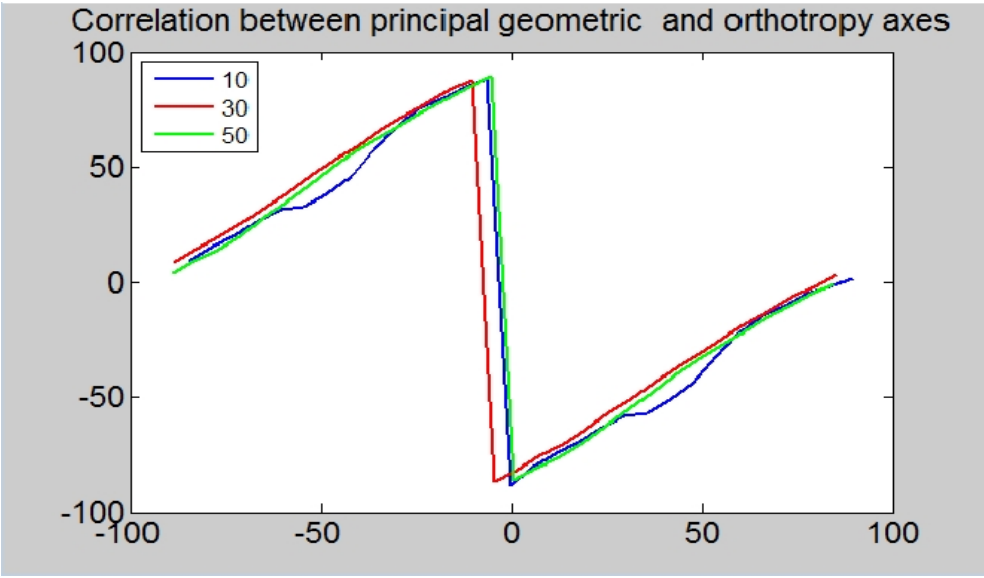

Figure 4.5: correlation between geometric and orthotropic axes for Experiment A, geometry of pores are ellipses $(\mathrm{a}=0.04, \mathrm{~b}=0.02)$ and cloud of porosity dimension is: $(0.8 \times 0.15)$ 
for $k_{11}$ and $k_{22}$ are inverse which show the fact that by changing the porosity orientation, when the thermal conductivity increases in $\mathrm{x}$ direction, it will decrease in $\mathrm{y}$ direction and vice versa.

In addition, these figures show higher ranges of thermal conductivity for higher porosities. For example in Fig 4.4, ranges of $k_{12}$ is between 0.0006 and 0.0032 when number of pores are 50 (porosity coefficient $=0.028$ ), but it is between 0.00048 and 0.0013 when number of pores are 30 (porosity coefficient $=0.018$ ). Same thing can be seen in Fig 4.3, ranges of $k_{22}$ is between 0.96 and 0.97 for 50 number of pores while it is between 0.9755 and 0.98 for 30 number of pores. It can be interpreted that when we have larger porosity coefficients, we should expect larger changes in amount of thermal conductivity regarding to the changes of the orientation of the pores.

Fig 4.4 shows that regardless of changes of orientation, the off diagonal elements of thermal conductivity tensor $\left(k_{12}\right)$, is very close to zero for materials having smaller amount of porosity or those which can be considered as homogeneous materials. With increasing the porosity coefficient, $k_{12}$ will increase and show more variation regarding to orientation changes as well. With a closer look to this graph, we can find that, the intersections of graphs are not exactly coinciding. It can be related to the random distribution of the pores which results in tiny differences in orientation of the porosity. [Please see Fig. 4.1].

In Fig 4.5, we could track changing of the anisotropy of thermal conductivity with respect to orientation of the pores (principal geometric axes). It can be seen that, there is a strong correlation between them and only small deviation can be found when number of ellipses are equal to 10 .

In the following, rest of the experiments are presented. In general, the trends in all the graphs are almost the same, but in case of observing any special case, it will be remarked.

\subsection{Experiment B}

In the next two experiments, we are going to investigate role of porosity coefficient. So we run the same test as experiment A, but only change dimensions of ellipses: $\mathrm{a}=$ 
$0.1, \mathrm{~b}=0.05$. Keeping these parameters untouched, we run the code for three different number of circles, 10, 30, 50 separately. The porosity coefficient in these three case is: 0.037, 0.082, 0.106. Results are plotted in Fig 4.7.

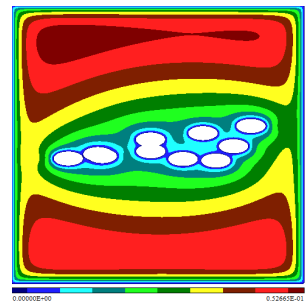

(a)

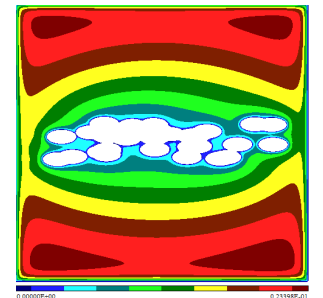

(b)

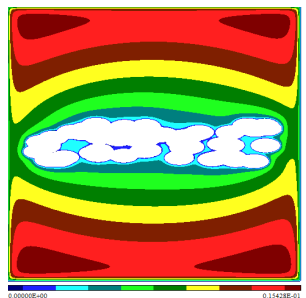

(c)

Figure 4.6: Cloud of porosity for experiment $\mathrm{B}$, geometry of pores are ellipses ( $\mathrm{a}=0.1$, $\mathrm{b}=0.05)$ and cloud of porosity dimension is: $(0.8 \times 0.15)$

\subsection{Experiment $\mathrm{C}$}

This experiment is the same as previous one, only we select bigger ellipses. Dimension of ellipses in this test is selected to be: $\mathrm{a}=0.2, \mathrm{~b}=0.1$. Also the dimension of the cloud of porosity is fixed to $(0.8 \times 0.15)$. Keeping these parameters untouched, we run the code for three different number of circles, 10, 30, 50 separately. The porosity coefficient for these cases are: 0.11, 0.168, 0.185. Results are plotted in Fig 4.9.

\section{Remark: The same trend as was explained in the observation part is seen in graph of these experiments.}

\subsection{Experiment D}

The goal of next two experiment is to investigate the effect of density of the distribution of the pores. We select the same parameters as experiment B, but using bigger cloud of porosity in order to decrease density of pores distribution. So, the geometry of the pores is selected to be ellipse with the these dimensions: $a=0.1, b$ $=0.05$. But dimension of cloud of porosity is changed to $(0.8 \times 0.4)$. Keeping these parameters untouched, we run the code for three different number of circles, 10, 30, 50 separately. In this case the porosity coefficients are: $0.038,0.101,0.147$. Result of this experiment can be seen in Fig 4.11. 


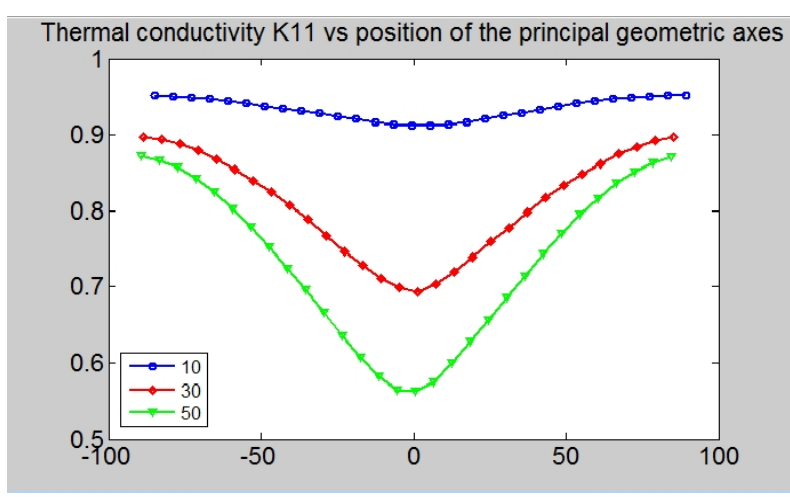

(a)

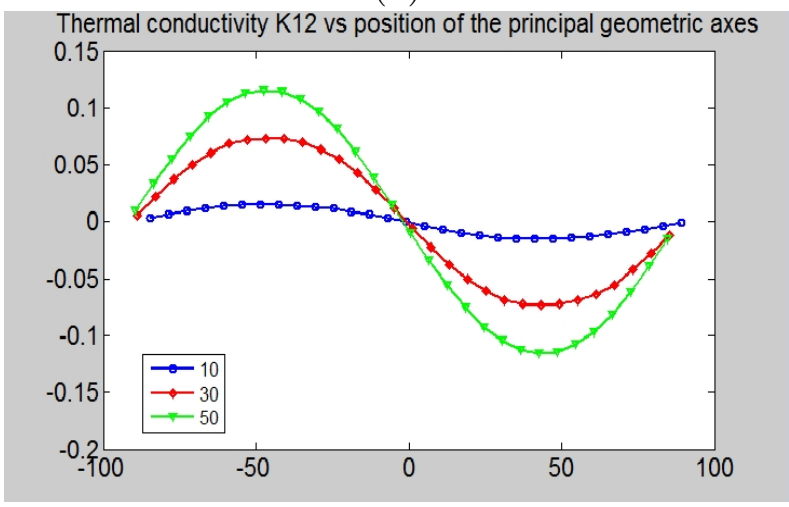

(c)

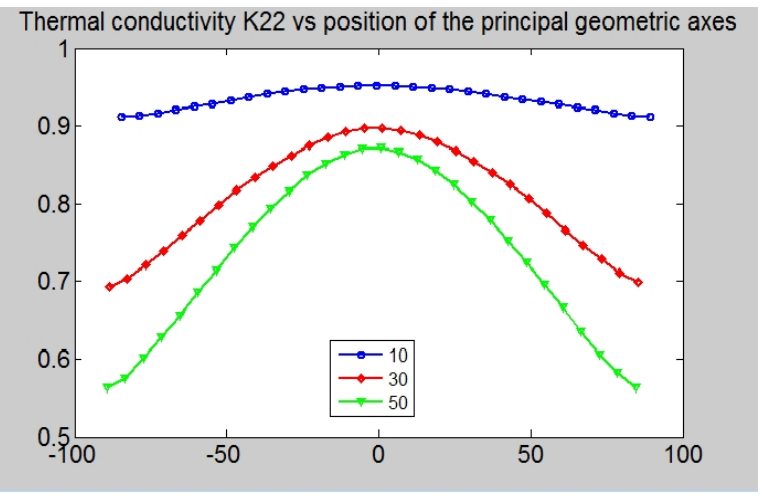

(b)

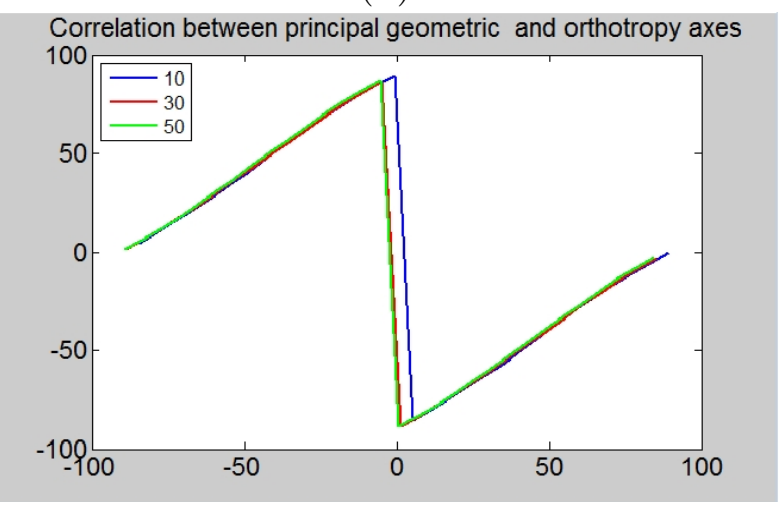

(d)

Figure 4.7: Results for Experiment B, geometry of pores are ellipses $(\mathrm{a}=0.1, \mathrm{~b}=0.05)$ and cloud of porosity dimension is: $(0.8 \times 0.15)$

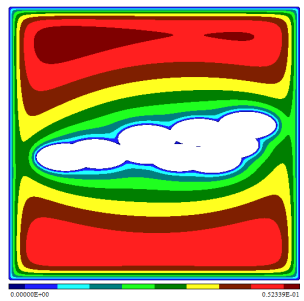

(a)

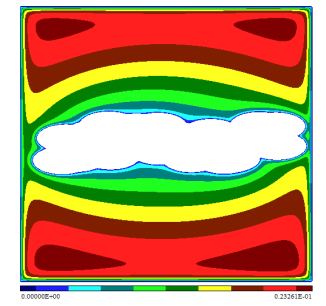

(b)

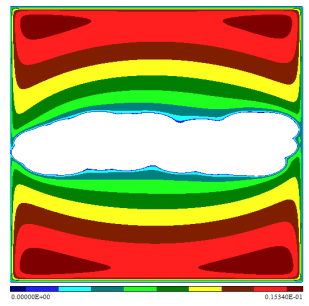

(c)

Figure 4.8: Cloud of porosity for experiment $\mathrm{C}$, geometry of pores are ellipses $(\mathrm{a}=0.2$, $\mathrm{b}=0.1)$ and cloud of porosity dimension is: $(0.8 \times 0.15)$ 


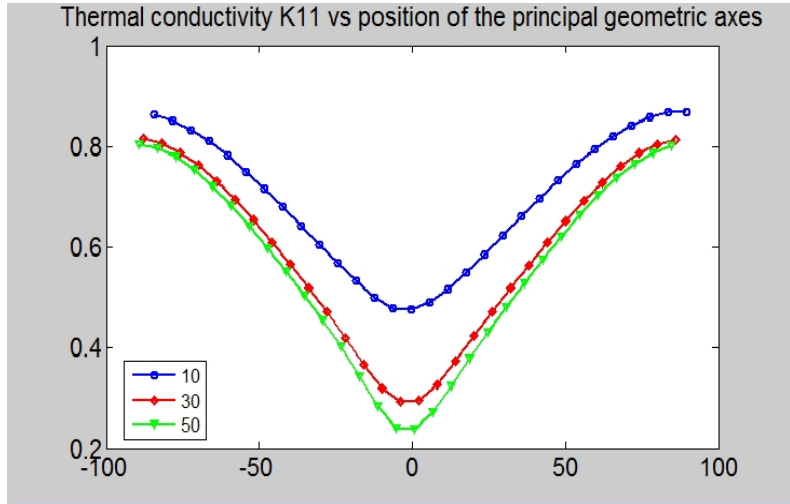

(a)

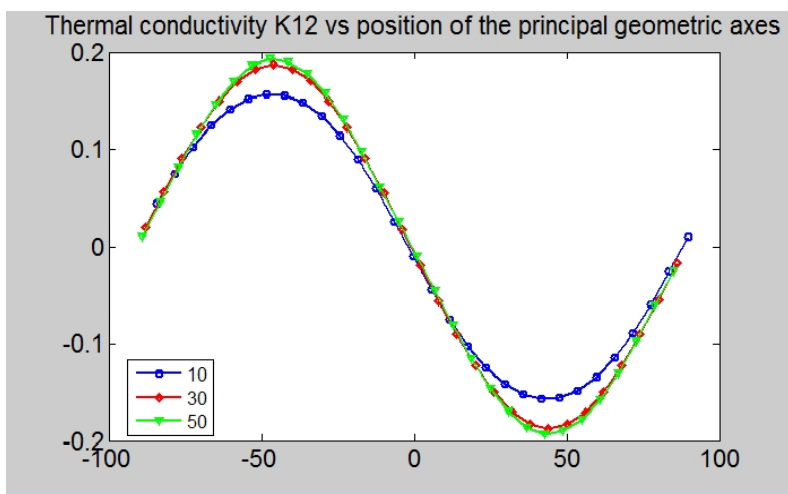

(c)

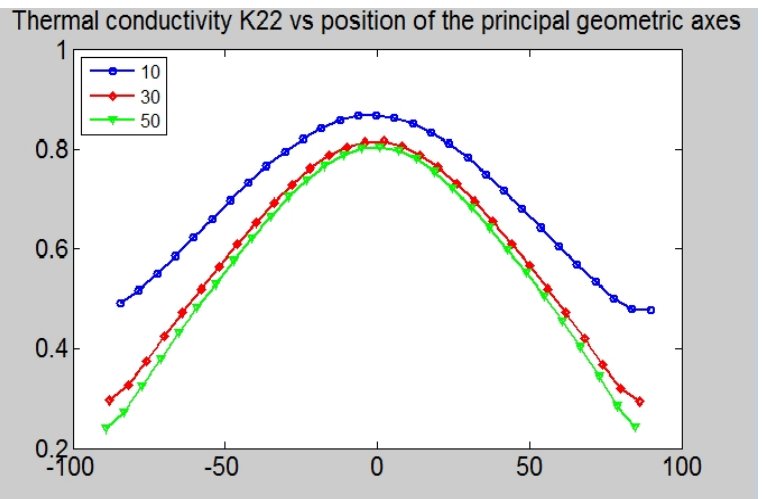

(b)

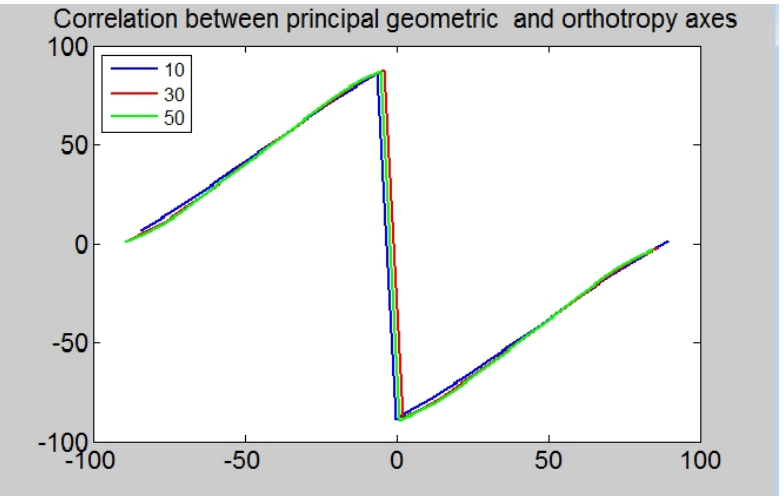

(d)

Figure 4.9: Results for Experiment $\mathrm{C}$, geometry of pores are ellipses $(\mathrm{a}=0.2, \mathrm{~b}=0.1)$ and cloud of porosity dimension is: $(0.8 \times 0.15)$

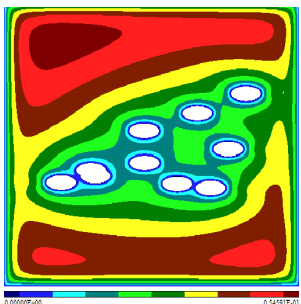

(a)

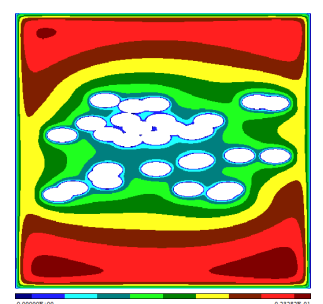

(b)

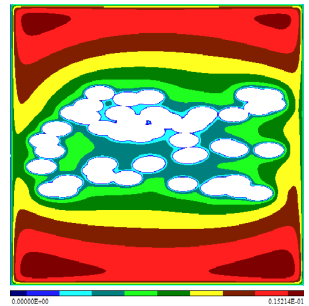

(c)

Figure 4.10: Cloud of porosity for experiment D, geometry of pores are ellipses $(\mathrm{a}=0.1$, $\mathrm{b}=0.05)$ and cloud of porosity dimension is: $(0.8 \times 0.4)$ 


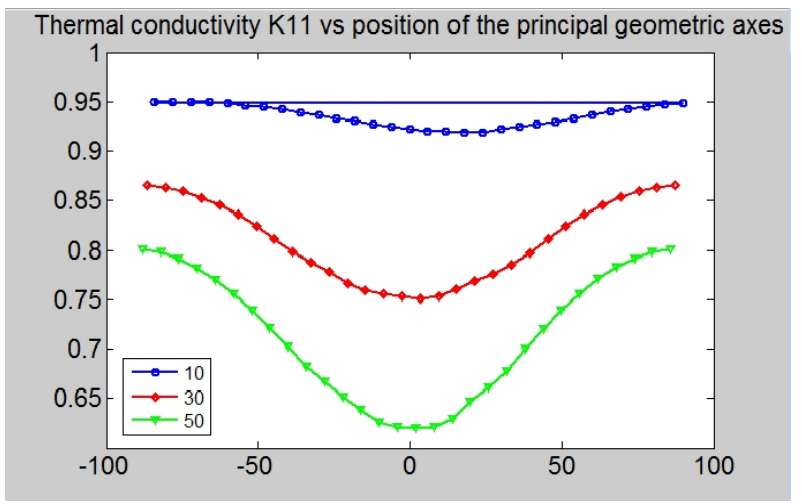

(a)

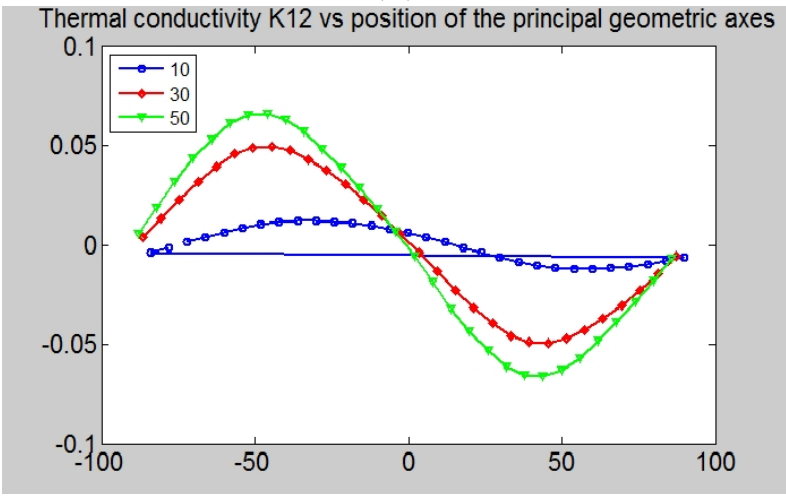

(c)

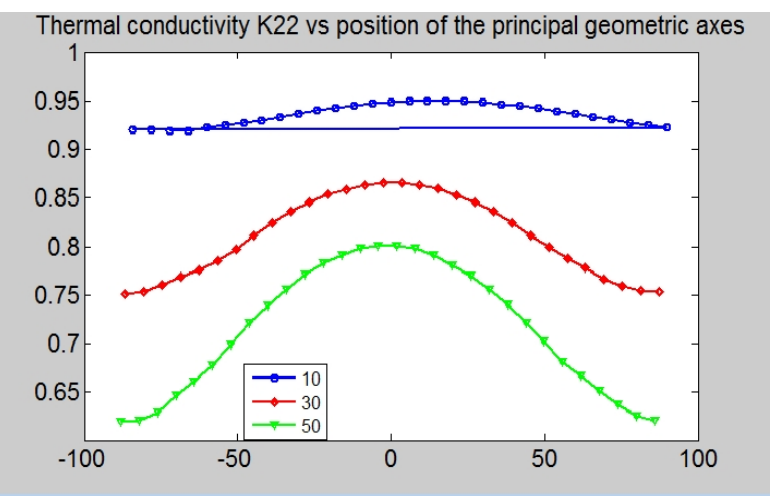

(b)

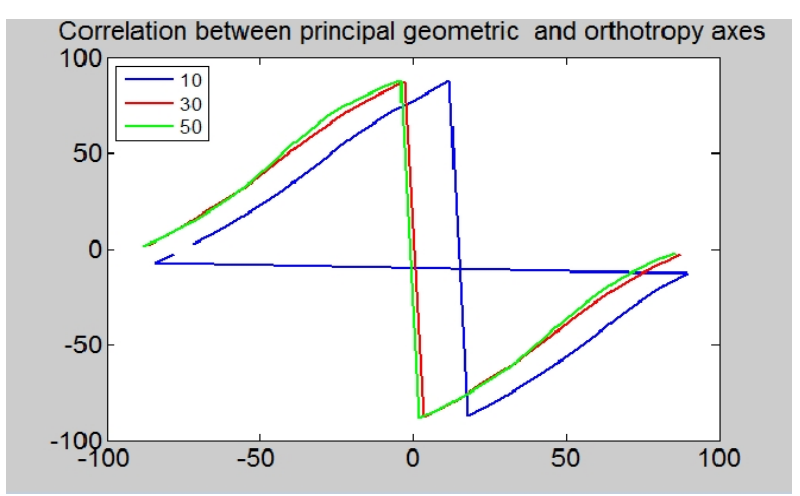

(d)

Figure 4.11: Results for Experiment D, geometry of pores are ellipses $(\mathrm{a}=0.1, \mathrm{~b}=$ $0.05)$ and cloud of porosity dimension is: $(0.8 \times 0.4)$ 


\subsection{Experiment E}

In this experiment, we continue the same trend as previous one and only change the dimension of the cloud of porosity to $(0.8 \times 0.6)$. Keeping other parameters untouched, we run the code for three different number of circles, 10, 30, 50 separately. The porosity coefficient in these cases are: 0.039, 0.107, 0.161 and results are plotted in Fig 4.13.

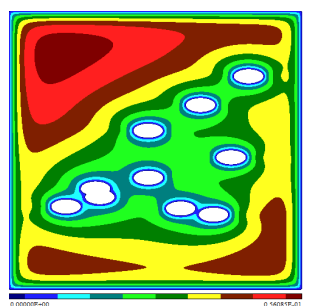

(a)

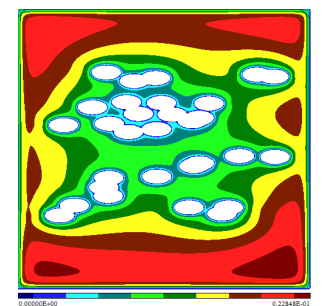

(b)

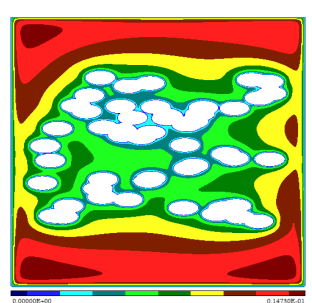

(c)

Figure 4.12: Cloud of porosity for experiment E, geometry of pores are ellipses $(\mathrm{a}=0.1$, $\mathrm{b}=0.05)$ and cloud of porosity dimension is: $(0.8 \times 0.6)$

\subsection{Experiment $\mathrm{F}$}

In the next five experiments, all the previous experiments will be tested on the circles as the geometry of the pores. This is done in order to investigate the effect of direction of the pores on the material behaviour in terms of thermal conductivity. (Ellipse is a kind of directional geometry while circles do not have direction).

Like Experiment A, B and C, cloud of porosity dimension is $(0.8 \times 0.15)$. Pores are circles with the radius of: $\mathrm{R}=0.02$. Keeping these parameters untouched, we run the code for three different number of circles, 10, 30, 50 separately. The porosity coefficient for these cases are: 0.004, 0.01, 0.015. The results can be found in Fig4.15

According to Fig 4.15, almost the same trend as previous ones is seen. Range of $k_{11}$ and $k_{22}$ for circular pores are less than ellipsoidal ones. Also there are more perturbation in last graph for the smallest porosity coefficient.

\subsection{Experiment G}




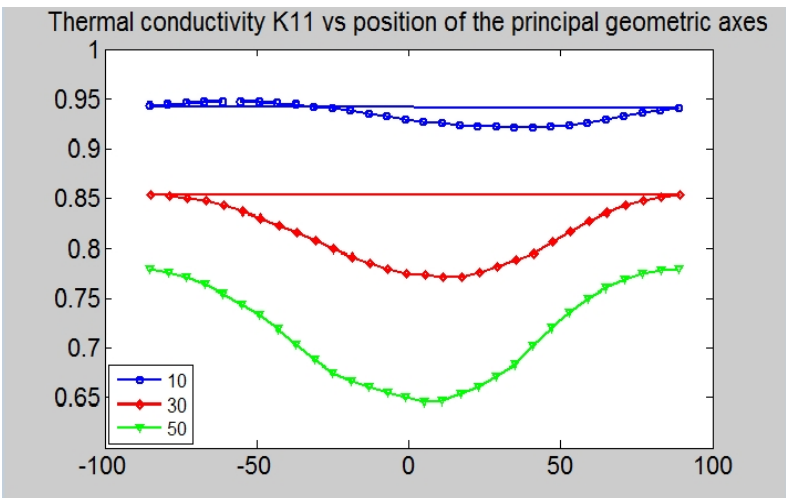

(a)

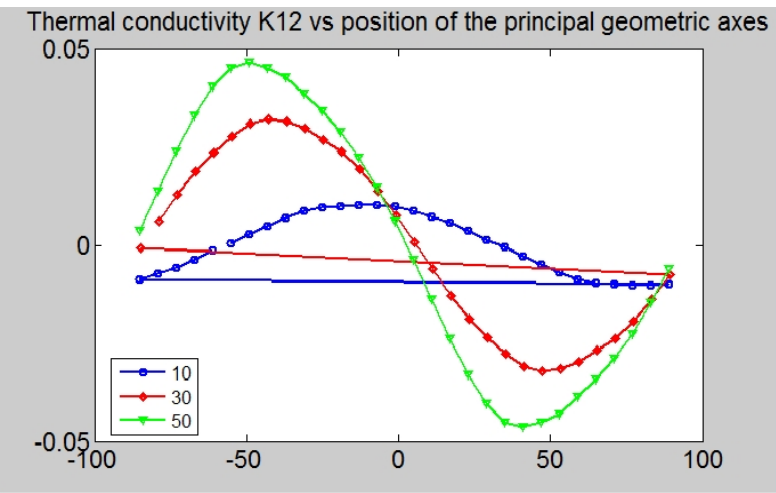

(a)

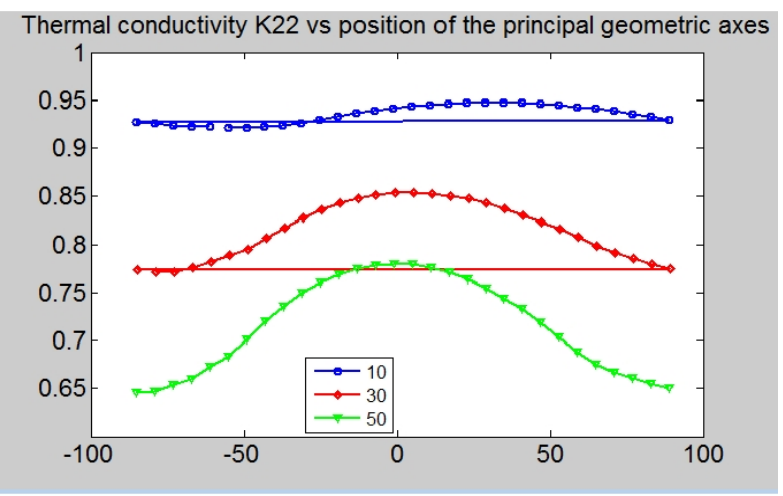

(b)

Correlation between principal geometric and orthotropy axes

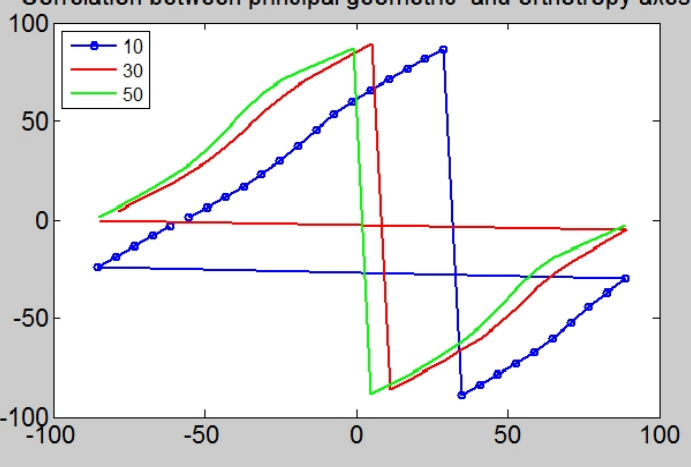

(b)

Figure 4.13: Results for Experiment E, geometry of pores are ellipses $(\mathrm{a}=0.1, \mathrm{~b}=0.05)$ and cloud of porosity dimension is: $(0.8 \times 0.6)$

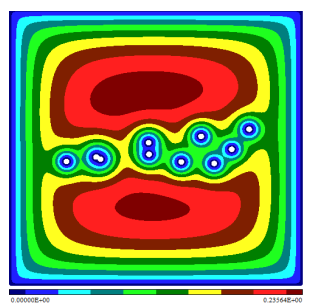

(a)

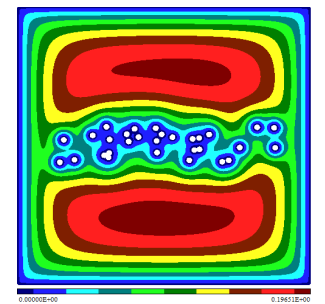

(b)

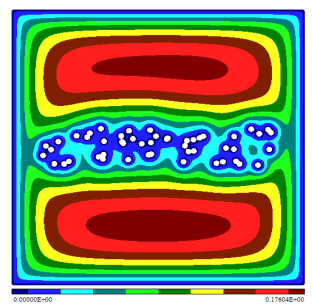

(c)

Figure 4.14: Cloud of porosity for experiment $\mathrm{F}$, geometry of pores are circles $(\mathrm{R}=$ $0.02)$ and cloud of porosity dimension is: $(0.8 \times 0.15)$ 


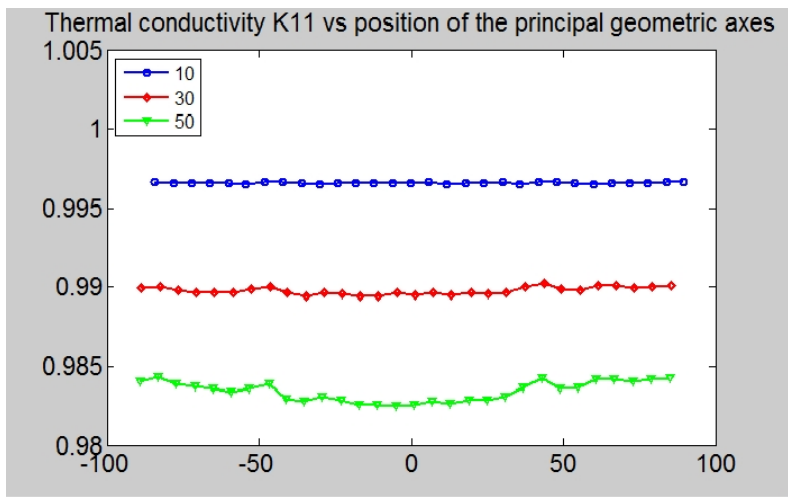

(a)

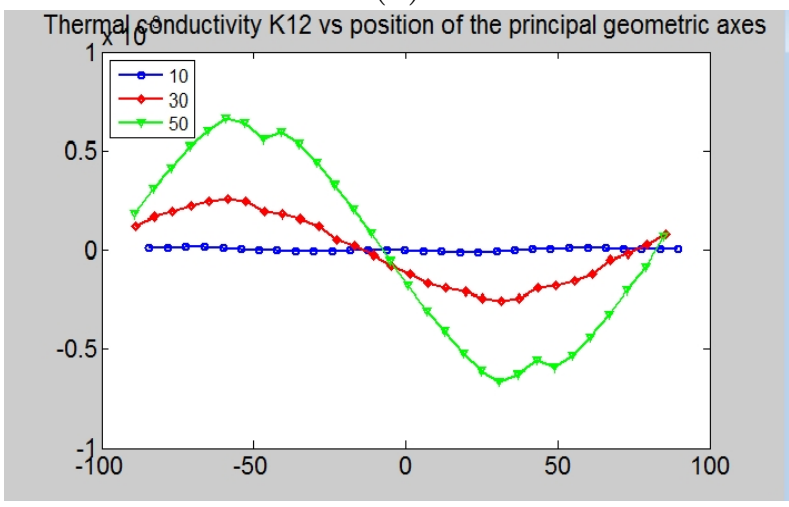

(c)

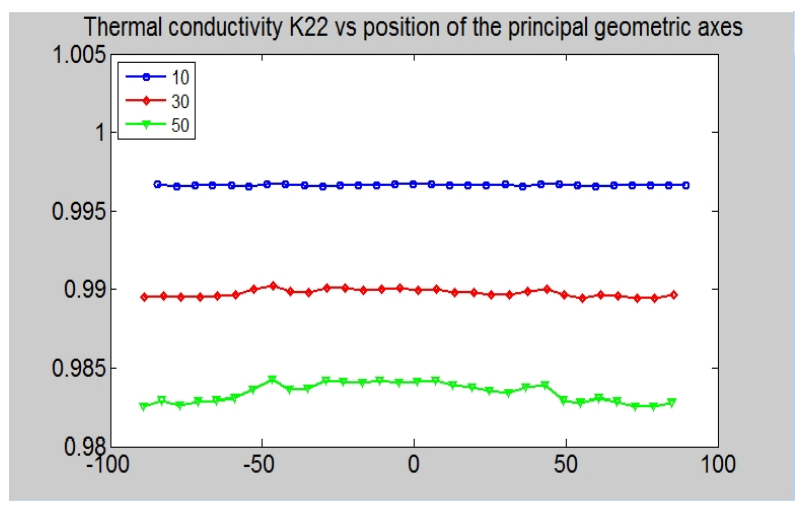

(b)

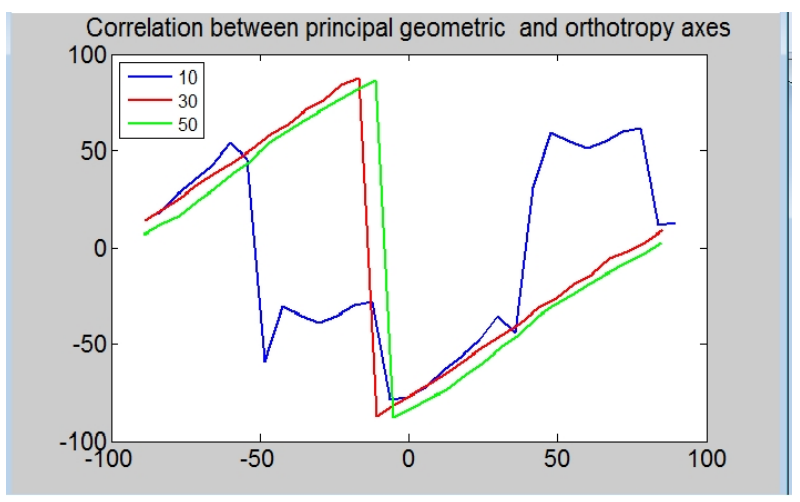

(d)

Figure 4.15: Results for Experiment $\mathrm{F}$, geometry of pores are circles $(\mathrm{R}=0.02)$ and cloud of porosity dimension is: $(0.8 \times 0.15)$ 
In this experiment, the radius of the pores is selected to be $\mathrm{R}=0.05$ in order to increase the porosity coefficient. The dimension of the cloud of porosity is unchanged: $(0.8 \times 0.15)$, similar to condition which was defined in experiment $F$. We run the code for three different number of circles, 10, 30, 50 separately. The porosity coefficients for these cases are: 0.019, 0.05, 0.071. Results can be seen in Fig 4.17.

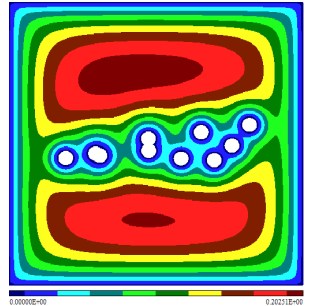

(a)

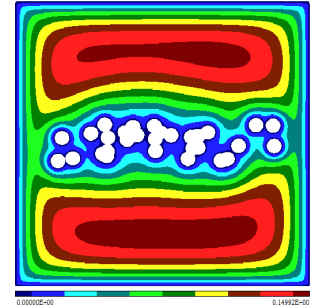

(b)

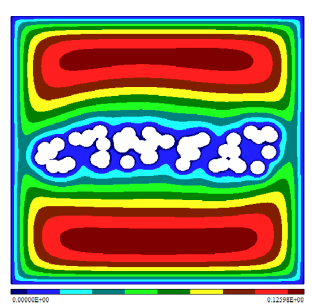

(c)

Figure 4.16: Cloud of porosity for experiment $G$, geometry of pores are circles $(R=$ $0.05)$ and cloud of porosity dimension is: $(0.8 \times 0.15)$

\section{Remark: There are very strong correlation between the assigned parameters and the graphs are smooth for both cases.}

\subsection{Experiment $\mathrm{H}$}

In this experiment, larger circles are selected: $\mathrm{R}=0.1$. The dimension of the cloud of porosity is unchanged: $(0.8 \times 0.15)$. The porosity coefficient in these cases increases to: $0.068,0.129,0.154$. The results of this part can be found in Fig 4.19.

\subsection{Experiment I}

In this experiment and the next one, the radius of pores is kept untouched, but we changed the dimension of cloud of porosity in order to check the effect of changing the density of distribution of the pores. Geometry of the pores is selected to be circle with radius of $\mathrm{R}=0.05$. Also the dimension of the cloud of porosity is selected to be: $(0.8 \times 0.4)$. We run the code for three different number of circles, 10, 30, 50 separately. The porosity coefficients for these cases are: $0.019,0.055,0.085$. The results are plotted in Fig 4.21. 


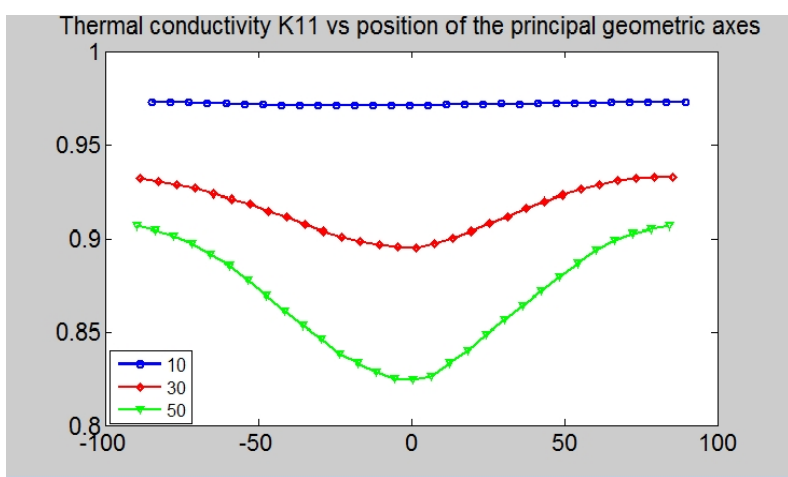

(a)

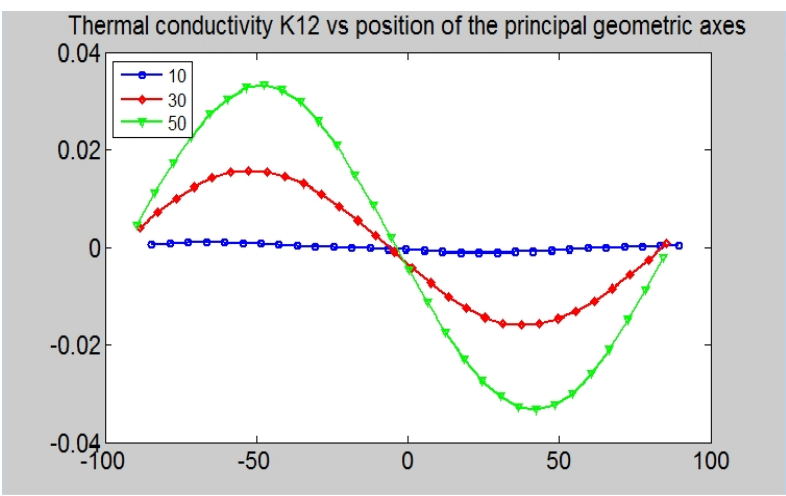

(c)

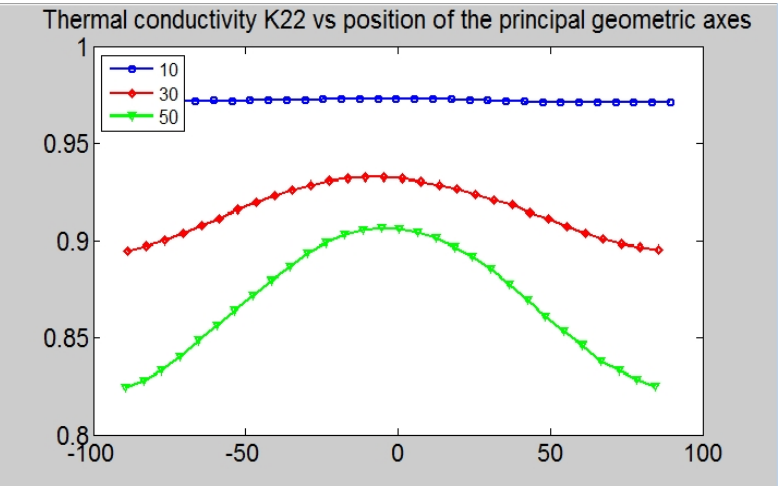

(b)

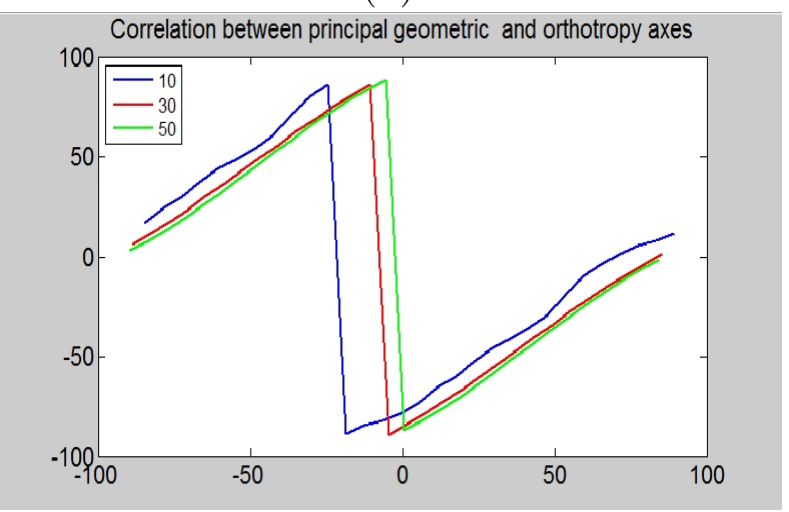

$(\mathrm{d})$

Figure 4.17: Results for Experiment $\mathrm{G}$, geometry of pores are circles $(\mathrm{R}=0.05)$ and cloud of porosity dimension is: $(0.8 \times 0.15)$ 


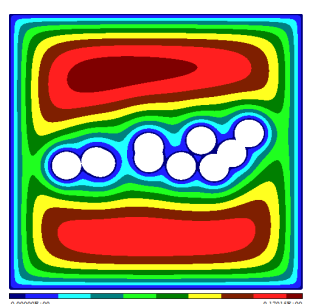

(a)

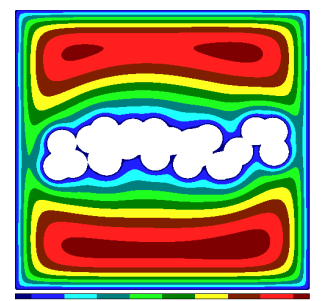

(b)

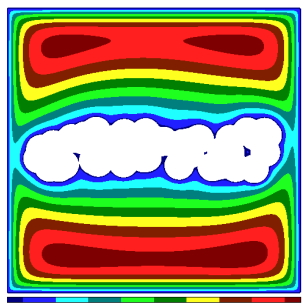

(c)

Figure 4.18: Cloud of porosity for experiment $\mathrm{H}$, geometry of pores are circles $(\mathrm{R}=0.1)$ and cloud of porosity dimension is: $(0.8 \times 0.15)$

Remark: As it is seen, lines which represent changes of the smallest porosity coefficient have more erratic changes.

\subsection{Experiment J}

As the last experiment, the dimension of the cloud of porosity is changed to $(0.8 \times 0.6)$. The geometry of the pores are circles with radius of $\mathrm{R}=0.05$. Keeping these parameters untouched, we run the code for three different number of circles, 10, 30, 50 separately. The porosity coefficient for these cases are: 0.02, 0.056, 0.089. Results can be found in Fig 4.23 .

Remark: There are more erratic changes in these figures. It can be due to the large cloud of porosity which is defined for this experiment. Changing the orientation of this cloud of porosity can produce this chaos, it means that, when pores are more disperse, rotating of the RVE will result in larger differences in thermal conductivity in each direction. Therefore it can be said that not only the porosity coefficient can affect the material behavior, but also the pores dispersion can be effective.

\subsection{Conclusion of the Experiments}

In experiments $\mathrm{A}, \mathrm{B}$ and $\mathrm{C}$, we gradually increase the porosity coefficient by increasing size of the pores and fixing the distribution density by keeping untouched the cloud of porosity dimension. We can see in Fig 4.24 that by increasing the porosity coefficient we will have smaller amount of thermal conductivity in every single angle of principal geometric axes. Meanwhile the off diagonal elements of thermal conductivity 


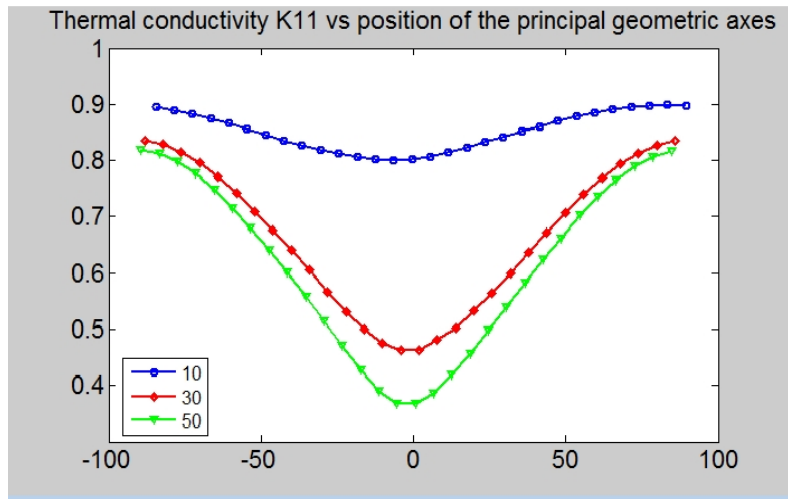

(a)

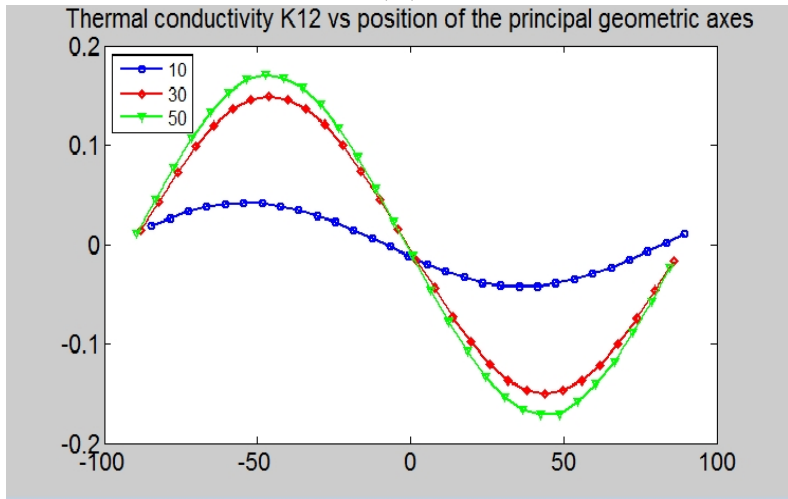

(c)

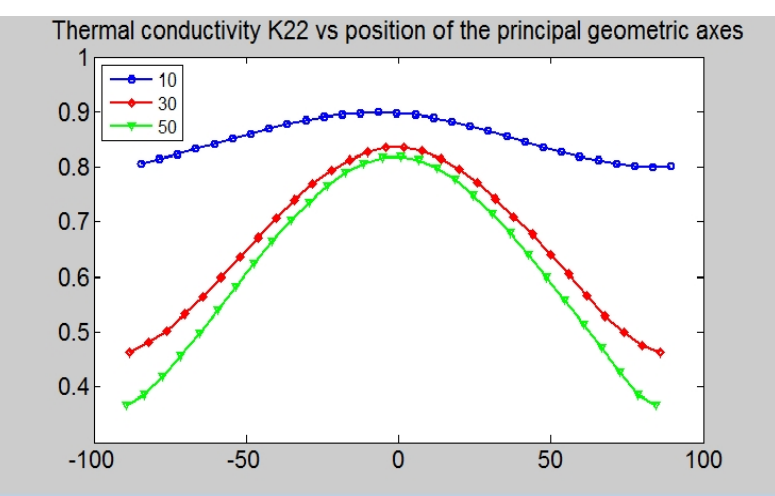

(b)

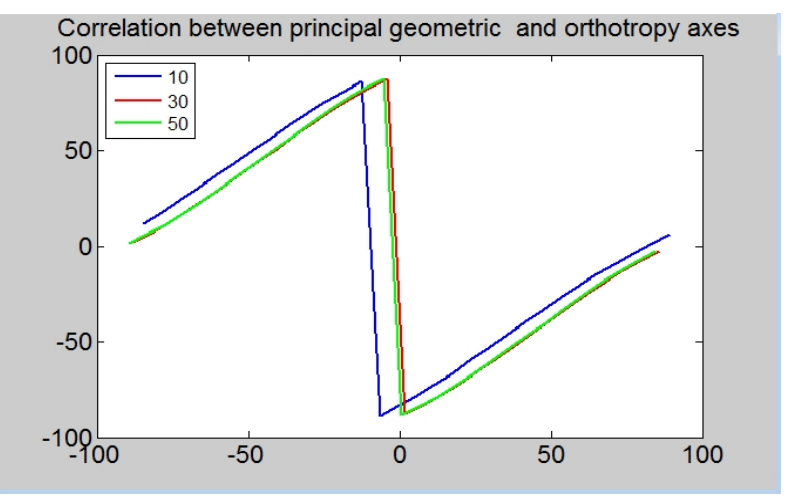

(d)

Figure 4.19: Results for experiment $\mathrm{H}$, geometry of pores are circles $(\mathrm{R}=0.1)$ and cloud of porosity dimension is: $(0.8 \times 0.15)$

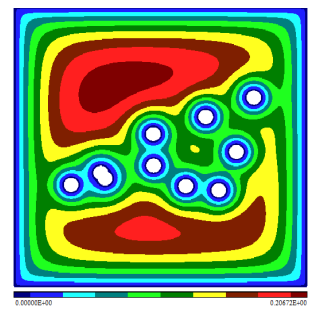

(a)

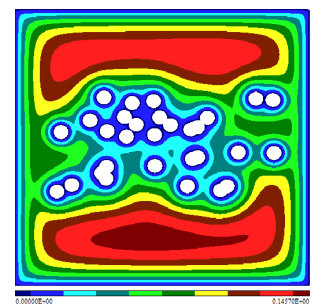

(b)

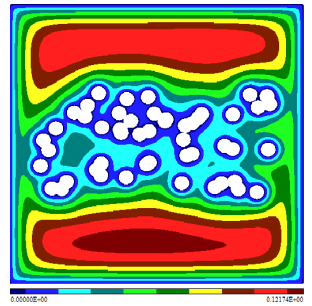

(c)

Figure 4.20: Cloud of porosity for experiment I, geometry of pores are circles $(\mathrm{R}=0.05)$ and cloud of porosity dimension is: $(0.8 \times 0.4)$ 


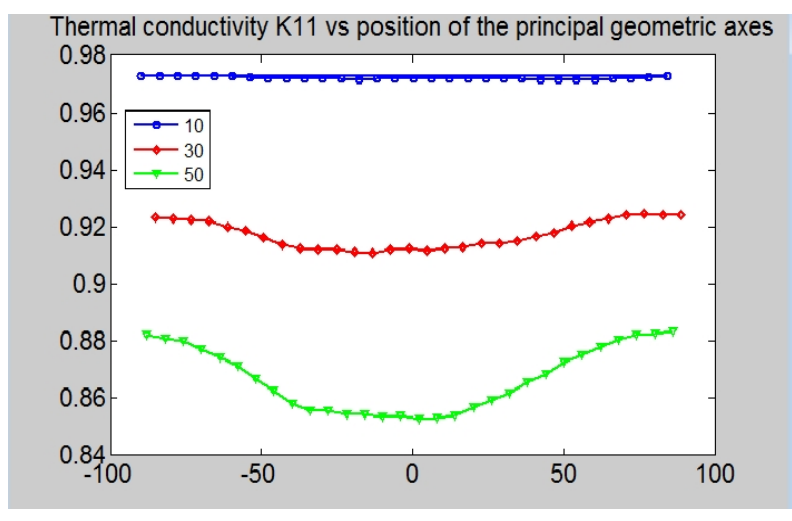

(a)

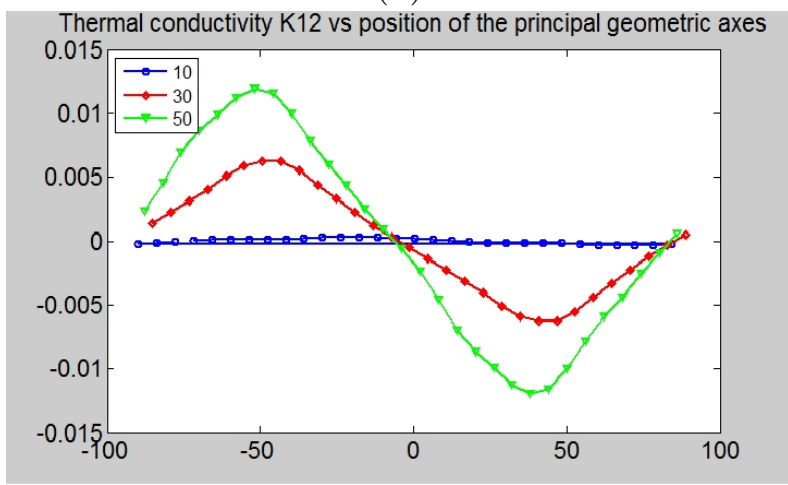

(c)

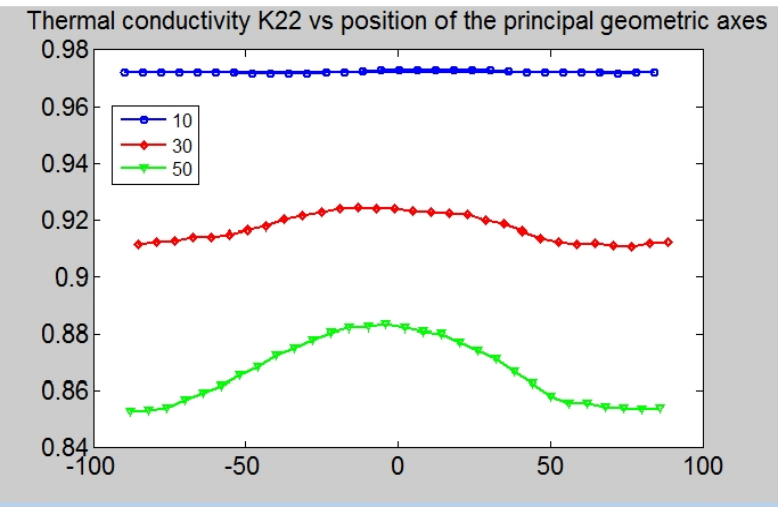

(b)

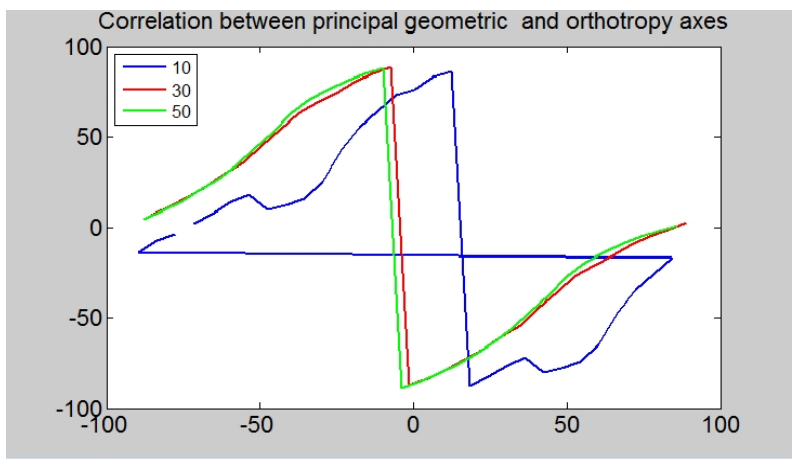

(d)

Figure 4.21: Results for Experiment I,geometry of pores are circles $(\mathrm{R}=0.05)$ and cloud of porosity dimension is: $(0.8 \times 0.4)$

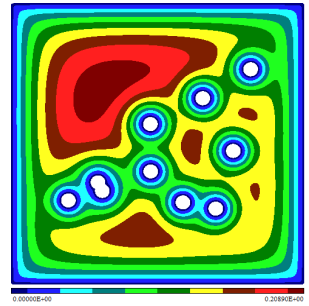

(a)

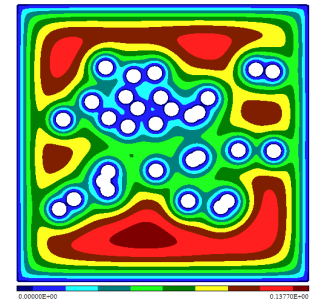

(b)

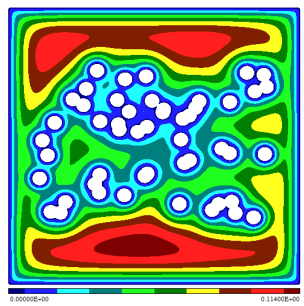

(c)

Figure 4.22: Cloud of porosity for experiment J, geometry of pores are circles $(\mathrm{R}=0.05)$ and cloud of porosity dimension is: $(0.8 \times 0.6)$ 


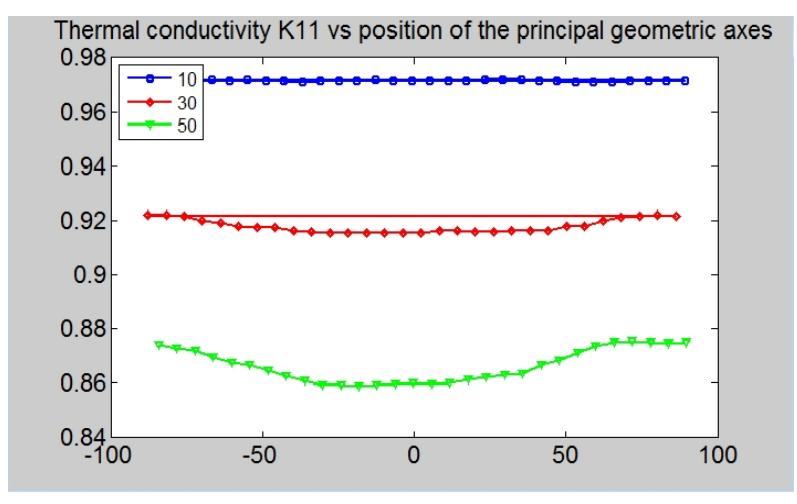

(a)

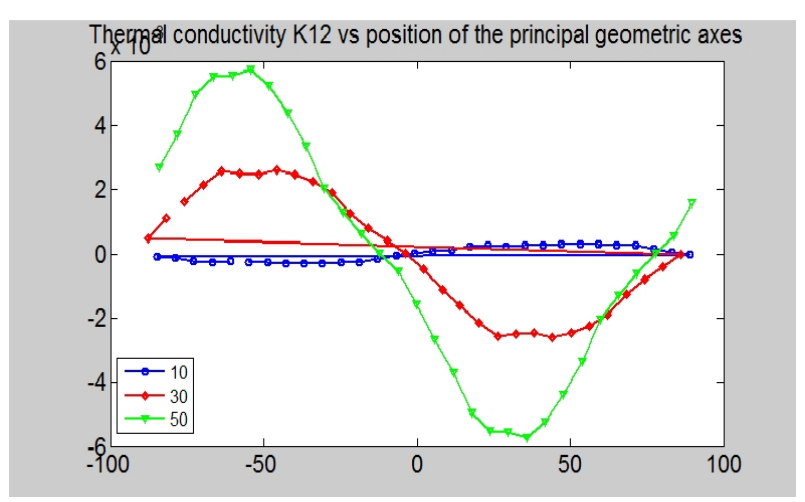

(c)

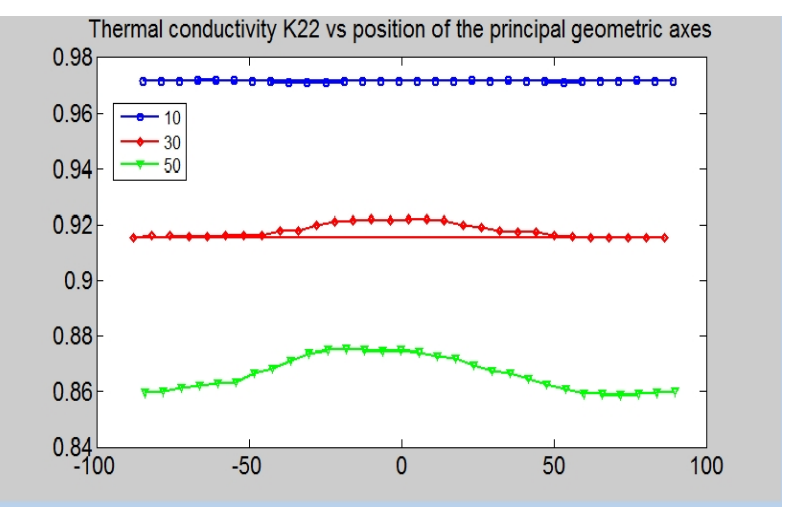

(b)

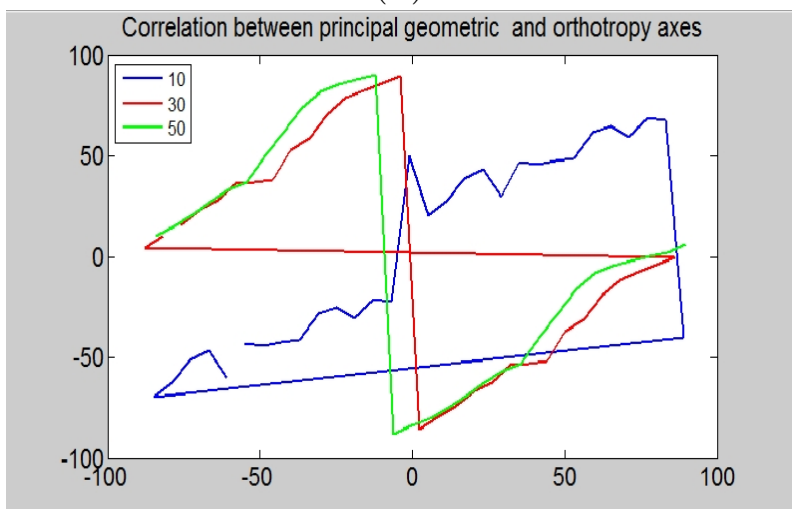

(d)

Figure 4.23: Results for Experiment J, geometry of pores are circles $(\mathrm{R}=0.05)$ and cloud of porosity dimension is: $(0.8 \times 0.6)$ 
$k_{12}$, has both increasing and decreasing trends, depend on the angle of principal geometric axes. It means that the trends depends on the orientation of the porosity. The same trend can be seen for experiment $\mathrm{G}, \mathrm{H}$ and I, when the geometry of pores is circle.

Also, for geometry of pores, we selected ellipses and circles in order to consider effect of pores shape on the material behavior. For clear investigation of this issue, we select results for the same porosity coefficient and also the same cloud of porosity dimension and plotted the results for both geometries in one graph. Fig 4.25 shows differences very well. We can see that with the same porosity coefficient (0.018) elements of thermal conductivity tensor for both shape are almost the same. It shows that shape of porosity can affect the homogenized thermal conductivity but the effect is not significant.

Dispersion of the pores is another parameter which was investigated in this chapter. In order to have clear conclusion about this parameter, we choose the results of experiment B, D and F for 30 numbers of ellipses and plotted them in Fig 4.26. In these experiments geometry and dimension of the pores are selected to be the same, ellipses (a $=0.1$ and $\mathrm{b}=0.05)$, but we gradually decrease pores distribution density by increasing the dimension of cloud of porosity in this way: $(0.8 \times 0.15),(0.8 \times 0.4),(0.8 \times 0.6)$. According to Fig. 4.26, when pores are more disperse, range of changes in element of thermal conductivity tensor regard to the angle of anisotropy are smaller. In this case, material microstructure behaviour goes toward more homogeneity, therefore, it can be less affected by changes of anisotropy. Also according to Fig 4.26, by decreasing the pores distribution density, no constant increasing or decreasing trend can be seen in the amount of thermal conductivity tensor elements and it depends on the orientation of the pores. 


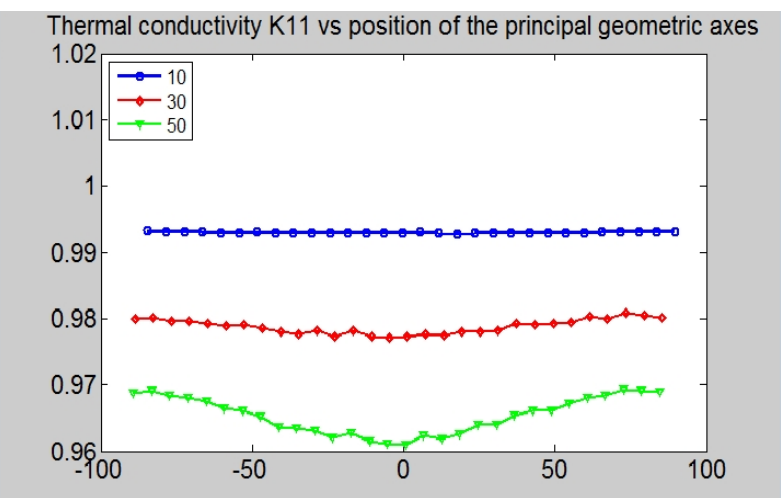

(a)

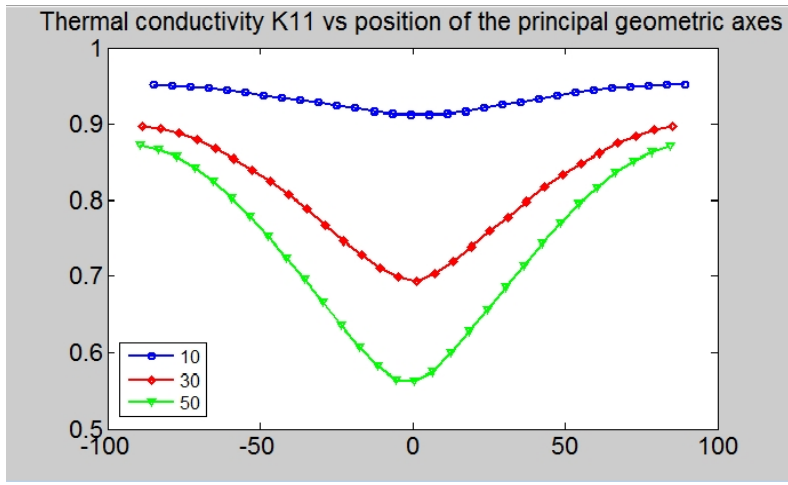

(c)

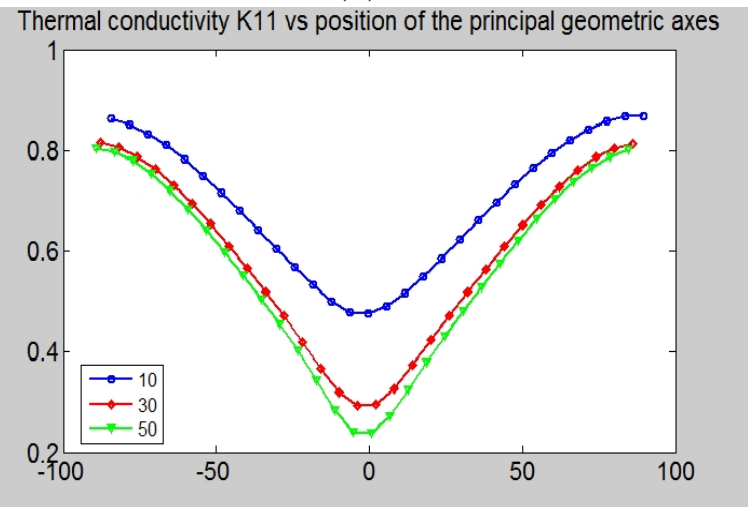

(e)

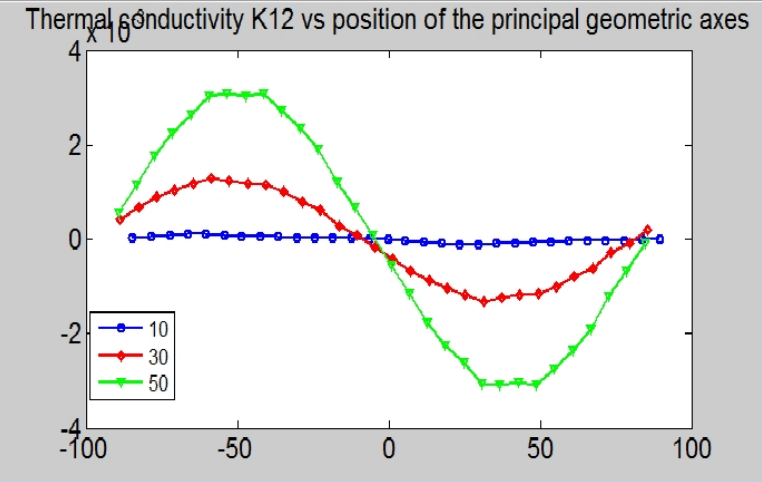

(b)

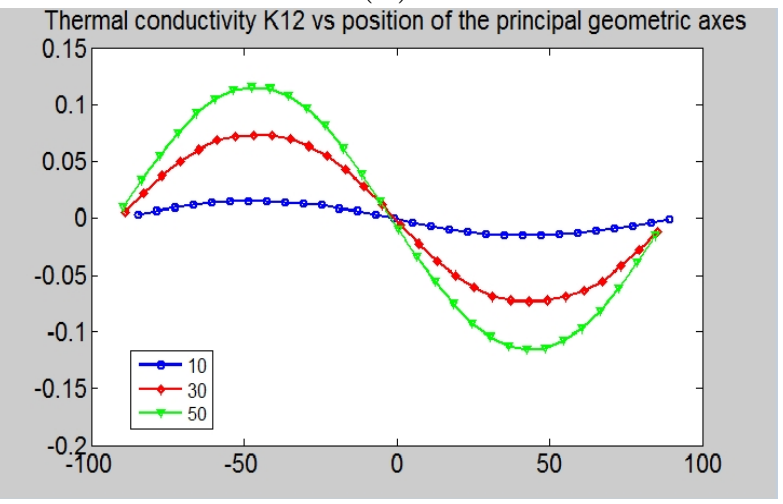

(d)

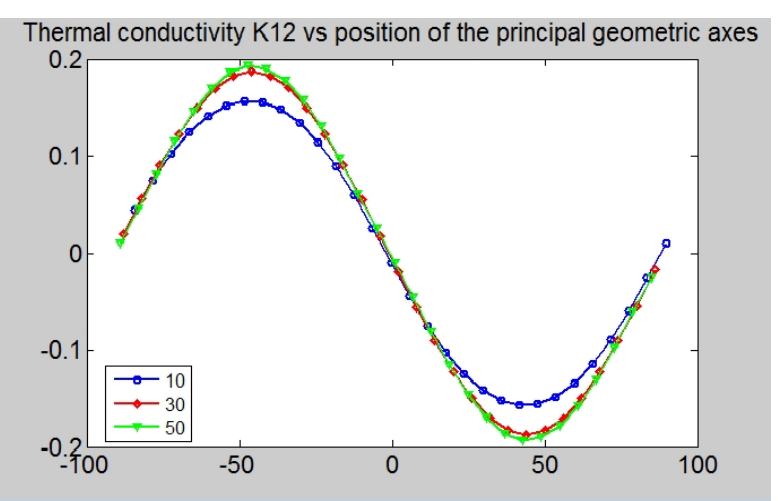

(f)

Figure 4.24: Comparison of the results by increasing the porosity coefficient, (a)and (b): $k_{11}$ and $k_{12}$ for porosity coefficients: $0.006,0.018,0.028$. (c) and (d): $k_{11}$ and $k_{12}$ for porosity coefficients: $0.037,0.082,0.106$. (e) and (f): $k_{11}$ and $k_{12}$ for porosity coefficients: $0.11,0.168,0.185$. 


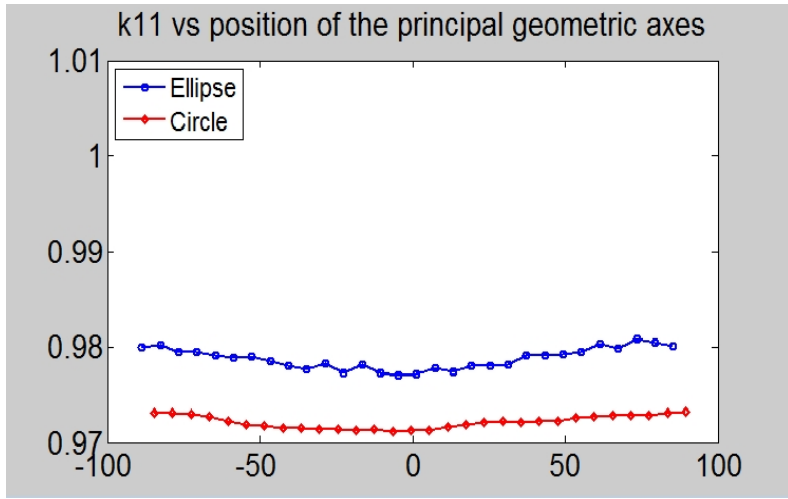

(a)

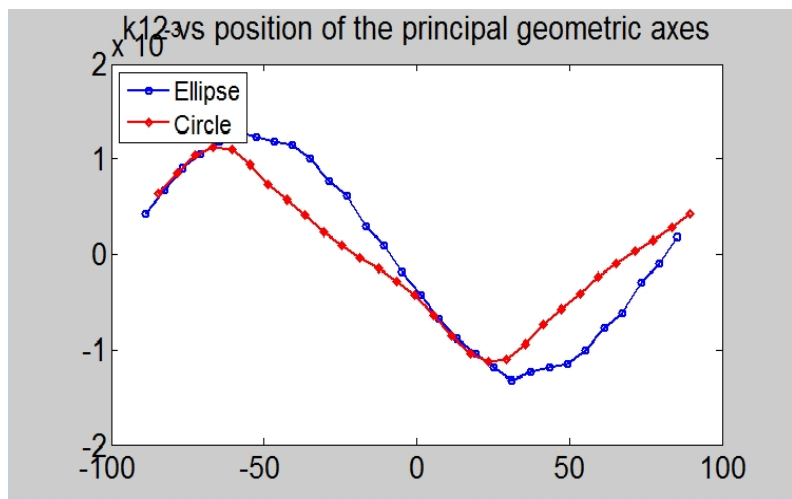

(c)

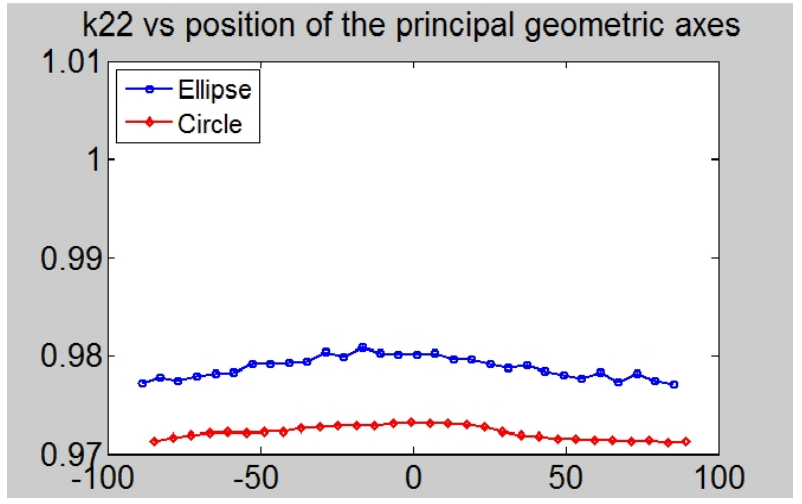

(b)

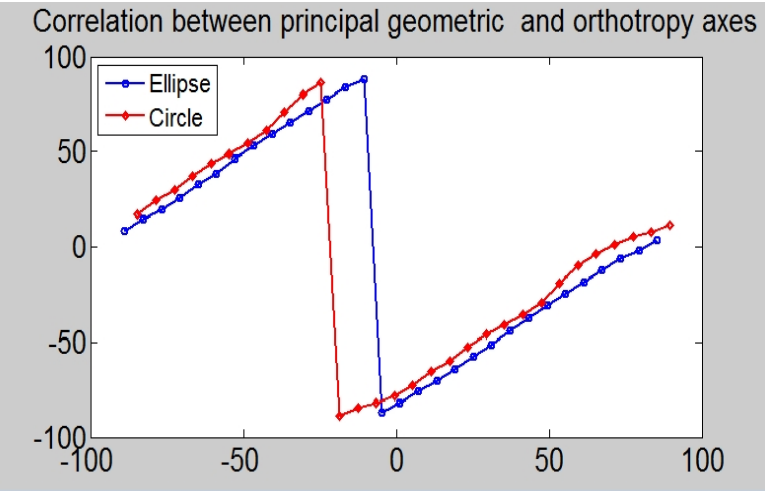

(d)

Figure 4.25: Comparison of the results of two different pore geometry 


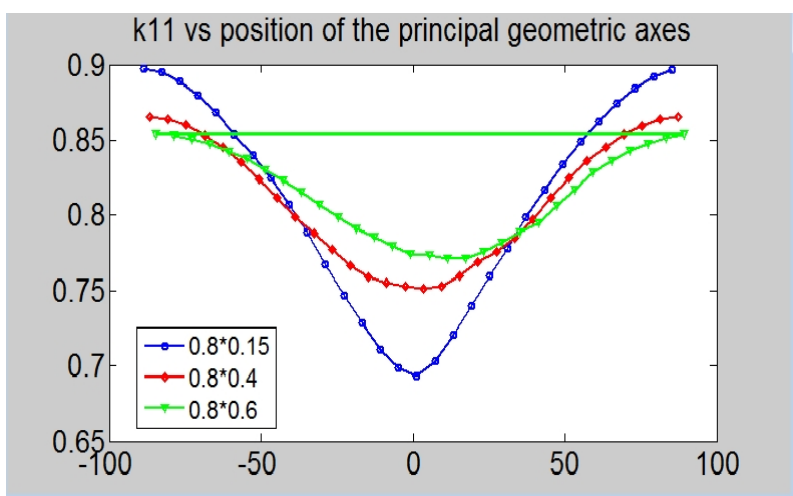

(a)

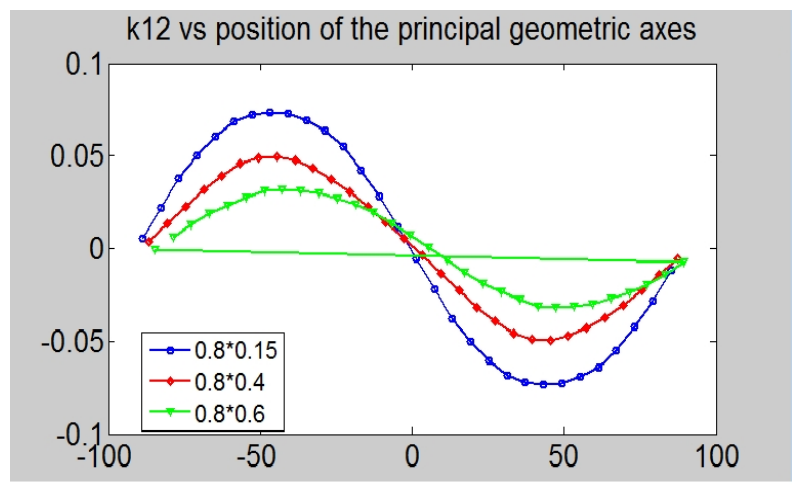

(c)

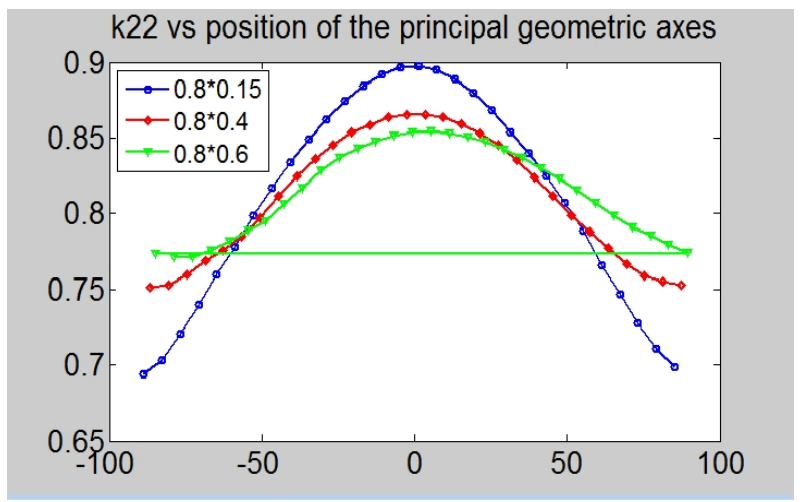

(b)

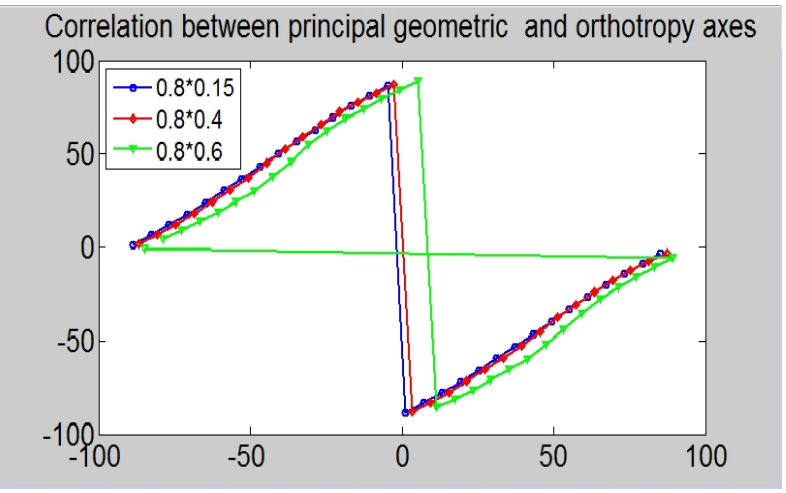

(d)

Figure 4.26: Comparison of the results for different pores distribution 


\section{CHAPTER 5 CONCLUSIONS}

In this thesis, we demonstrated the feasibility of homogenization of thermal properties of porous ceramic materials using meshfree method with distance field. We showed how to combine asymptotic homogenization and meshfree method for finding thermal conductivity. We presented results of several example and investigated the effect of anisotropy in elements of thermal conductivity tensor. It is learned that the geometry of the microstructure affected the anisotropy of the thermal conductivity.

Since porous microstructure of the ceramic materials highly affects the macroscopic materials properties, the geometry of the microstructure has to be included into consideration during the analysis. Direct inclusion of the geometry of the microstructure into analysis at the macro level is infeasible due to excessive computational cost. That is why homogenization method was applied.

Homogenization requires solution of the boundary value problem at micro scale level. Mesh-based analysis methods, like Finite Element Method, require spatial meshing for solving boundary value problem. Meshing of the realistic geometry of the microstructure is difficult due to data conversion from 2D images and Computed Tomography (CT)scans. Applying meshfree method which does not need to construct the grid which must conform to the geometry, solved problems of meshing and enabled us to include realistic geometry of the microstructure to homogenization procedure.

Further more, dependence of the homogenized thermal conductivity to the geometry of the microstructure was investigated and following points were concluded:

- Porosity coefficient affects homogenized material properties

- Rule of mixture cannot predict anisotropy of the homogenized material properties

- Shape and distribution of the pores may result in anisotropy of the homogenized material properties

- Orientation of the principal axes of anisotropy is well correlated with the orientation of the principal axes of the porosity structure 
- Dispersion of pores affects homogenized material properties. Higher dispersion of the pores decreases anisotropy of the thermal conductivity

- Shape of the pores also affects the homogenized thermal conductivity 


\section{REFERENCES}

[1] A.Bhatia. Thermal Spraying Technology and applications. U.S Army Corps of engineers, Washangton DC 20314-1000, em 110-2-3401 edition, January 1999.

[2] HR. Argyris. Energy theorems and structural analysis. Aircraft Engineering.

[3] Cheung, c.zienkiewicz, and Y.K. The Finite Element Method in Structural and Continuum Mechanics. MCGrew Hill, 1967.

[4] Michael Freytag, Vadim Shapiro, and Igor Tsukanov. Field modeling with sampled distances. Computer Aided Design, 38:87-100, 2006.

[5] T. Kanit, S. Forest, I. Galliet, V. Mounoury, and D. Jeulin. Determination of the size of the representative volume element for random composites: statistical and numerical approach. solids and structure, 40, 2003.

[6] L. V. Kantorovich and V. I. Krylov. Approximate methods of higher analysis. Interscience Publishers, 1958.

[7] James K.Carson, Simon J.Lovatt, David J.Tanner, and Andrew C.Cleland. Thermal conductivity bounds for isotropic, porous materials. Heat and mass transfer, 48:2150-2158, 2005.

[8] B. Klusemann and B. Svendsen. Homogenization methods for multi-phase elastic composites: Comparisons and benchmarks. TECHNISCHE MECHANIK, 30(4):374 $-386,2010$.

[9] Srihari Kurkuri. A review of homogenization thechniques for heterogeneous materials. March 2004.

[10] Yasser M.Shabana and Naotaka Noda. Numerical evaluation of the thermomechanicaleffective properties of a functionally graded material using the homogenization method. Solid and Structures, 45:3494-3506, February 2008.

[11] Przemieniecki and J.S. Theory of Matrix Structural Analysis. MCGrew Hill, 1968.

[12] V. L. Rvachev. Theory of r-functions and some applications. Naukova Dumka, 1982 .

[13] V. L. Rvachev, T. I. Sheiko, V. Shapiro, and I. Tsukanov. On completeness of rfm solution structures. Computational Mechanics, 25:305-316, 2000.

[14] Rao Singiresus S. The Finite Element Method in Engineering. Elsevier, 2004.

[15] V. Shapiro and I. Tsukanov. Implicit functions with guaranteed differential properties. In Fifth ACM Symposium on Solid Modeling and Applications, pages 258-269, 1999.

[16] V. Shapiro and I. Tsukanov. Meshfree simulation of deforming domains. ComputerAided Design, 31(7):459-471, 1999. 
[17] Mohammadi Soheil. Extended Finite Element Method for Fracture Analysis of Structures, PUBLISHER $=$.

[18] Young Seok Song and Jae Ryoun Youn. Evaluation of effective thermal conductivity for carbon nanotube/polymer composites using control volume finite element method. Carbon, 44:710-717, November 2005.

[19] Jan Stransky, Jan Vorel, and michal Sejnoha. Mori- tanaka based estimates of effective thermal conductivity of various engineering materials. Micromechanics, 2:129-149, 2011.

[20] M.J Turner, R.W.Clough, H.C.Martin, and L.J.Topp. Stiffness and deflection analysis of complex structures. Aeronautical Sciense, 23:805-824, 1956.

[21] John B. watchman, editor. Ceramic innovations in the 20th century. 1999.

[22] Peter W.Chung, Kumar K.Tamma, and Raju R.Namburu. Asymptotic expansion homogenization for heterogeneous media. Composites, 32:1291-1301, June 2001.

[23] A. Zivelonghi, F. Cernuschi, C. Peyrega, D. Jeulin, S. Lindig, and J.H. You. Influence of the dual-scale random morphology on the heat conduction of plasma-sprayed tungsten via image-based fem. Computational Materials Science, 68:5-7, 2013. 